\title{
Parure, parfum, pavane : le regard genré et deux Madeleine de Carlo Crivelli
}

Finery, perfume, pavane: gendered gaze and two Mary Madgalens by Carlo Crivelli

\section{Vicki-Marie Petrick}

\section{OpenEdition}

\section{Journals}

Édition électronique

URL : http://journals.openedition.org/imagesrevues/7833

DOI : 10.4000/imagesrevues.7833

ISSN : 1778-3801

Éditeur :

Centre d'Histoire et Théorie des Arts, Groupe d'Anthropologie Historique de l'Occident Médiéval, Laboratoire d'Anthropologie Sociale, UMR 8210 Anthropologie et Histoire des Mondes Antiques

Référence électronique

Vicki-Marie Petrick, «Parure, parfum, pavane : le regard genré et deux Madeleine de Carlo Crivelli », Images Re-vues [En ligne], 16 | 2019, mis en ligne le 21 décembre 2019, consulté le 03 février 2021. URL : http://journals.openedition.org/imagesrevues/7833 ; DOI : https://doi.org/10.4000/ imagesrevues.7833

Ce document a été généré automatiquement le 3 février 2021.

Images Re-vues est mise à disposition selon les termes de la Licence Creative Commons Attribution Pas d'Utilisation Commerciale 4.0 International. 


\section{Parure, parfum, pavane : le regard genré et deux Madeleine de Carlo Crivelli}

Finery, perfume, pavane: gendered gaze and two Mary Madgalens by Carlo Crivelli

\section{Vicki-Marie Petrick}

Je tiens à remercier Gwladys LeCuff, Nicolas Sarzeaud, Guillaume Cassegrain et Thomas Golsenne pour leurs précieuses relectures, aussi bien qu'Antonio Montefusco, et Sylvain Piron pour leurs apports linguistiques. Un travail sur la Madeleine dans les Pietà de Crivelli attendra un autre article. 
Deux Marie-Madeleine, peintes par Carlo Crivelli entre 1470 et 1492 nous surprennent par leur regard qui croise celui du spectateur de façon bien inhabituelle : un regard en coin, un regard séducteur. Luxe ostentatoire et grâces maniérées - spécialités de ce peintre de la ligne gothique et des atours mondains viennent compléter la mise de ces images de la sainte pécheresse qui se serait convertie en oignant les pieds du Christ avec son parfum, ses larmes, les aurait

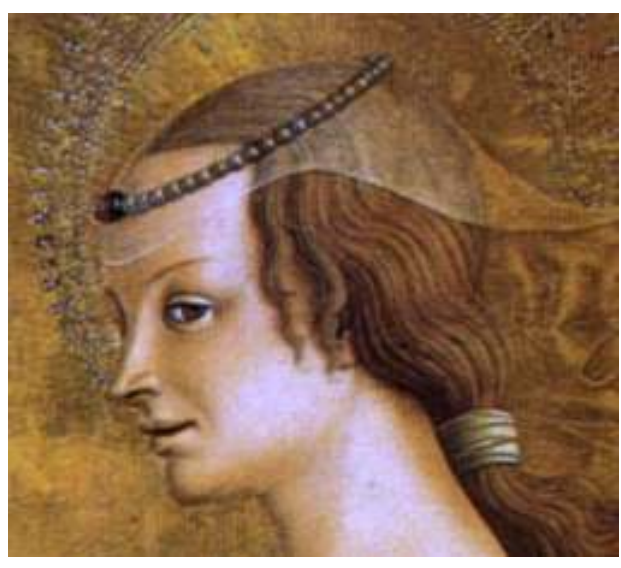
essuyés avec ses cheveux. Après avoir été son fidèle disciple pendant la Passion, elle fut récompensée en étant le premier témoin de la Résurrection. Après les événements bibliques, la légende apocryphe rapporte qu'elle aurait vécu au désert dans le dénuement et la privation, en extase quotidienne, habillée par ses seuls cheveux. Chacune de ces Madeleine de Crivelli se trouvait à l'origine dans une église reculée de l'Italie marchisane: Montefiore dell'Aso pour la première (fig. 1), Carpegna pour la deuxième (fig. 2), loin des grands foyers de la production artistique de l'Italie d'alors, tels Florence ou Venise. Après avoir quitté sa Venise natale - à cause d'un scandale d'enlèvement adultère ${ }^{1}$ - Crivelli eut à pratiquer sa profession dans ces Marches voisines, où un certain nombre de ses compères avait déjà laissé des traces de leur talent. Avec Iacobello da Fiore et Giovanni Bellini à Pesaro ou Gentile da Fabriano à Valleromita, par exemple, nous pourrions peut-être même parler d'un cercle artistique vénéto-marchisan ${ }^{2}$. 
Fig. 1

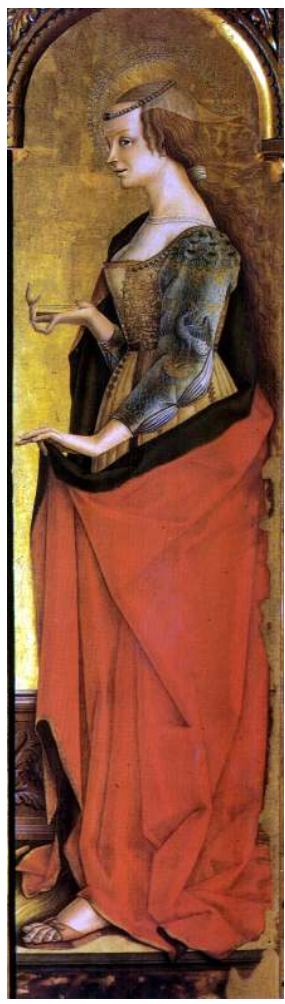

Carlo Crivelli, S. Marie-Madeleine, détail du Polyptyque de San Francesco de Montefiore, entre 1470 et 1473, tempera sur bois, $174 \times 54 \mathrm{~cm}$, Montefiore del'Aso, S. Lucia

Cliché Sailko, Wikimedia Commons

2 Là, Crivelli poursuivra une carrière marquée par le succès auprès des plus grandes familles de la région à travers la deuxième moitié du Quattrocento ${ }^{3}$. À la fin des années 1460, début des années 1470 , Crivelli reçoit la commande d'un polyptyque, aujourd'hui démembré, pour l'église des franciscains de Montefiore dell'Aso ${ }^{4}$. Il s'engage alors dans une réflexion sur l'image de la sainte qui est déjà, on le verra, étonnante. Puis vers 1492, le Vénitien reçoit une commande pour formuler à nouveau une version de la peccatrix. Il s'agit cette fois d'un panneau individuel et d'une Madeleine qui n'a jamais partagé la vedette avec d'autres saints. 
Fig. 2

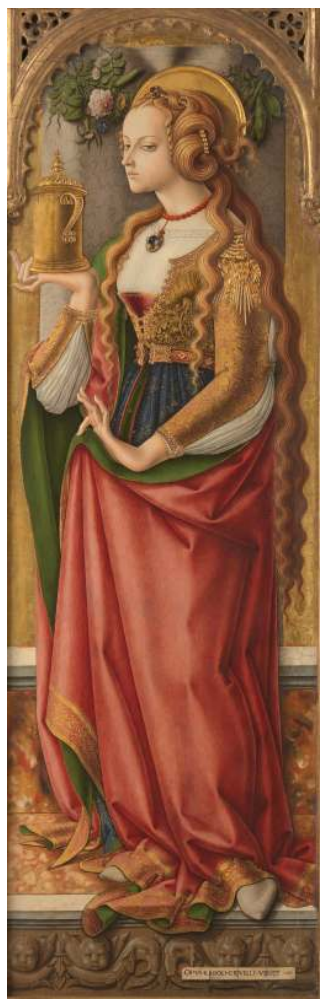

Carlo Crivelli, S. Marie-Madeleine, 1492, tempera sur bois, 152 × 49 cm, Rijsksmuseum, Amsterdam cliché Sailko, Wikimedia Commons

3 Ici Crivelli se souvient clairement de sa première version, mais les années écoulées lui ont apporté une vision plus précise et offert une élaboration plastique plus approfondie. Ni le caractère typiquement crivellesque de ces deux Madeleine ni leur apparition dans un environnement franciscain ne sont étonnants. La piété tout d'amour fervent de la Madeleine, si attachée à la Passion, était parfaitement en harmonie avec le charisme des disciples de François d'Assise. De plus, les frères mineurs ont été à la pointe de l'iconographie magdaléenne dès le Duecento sur leurs croix peintes. 
Fig. 3

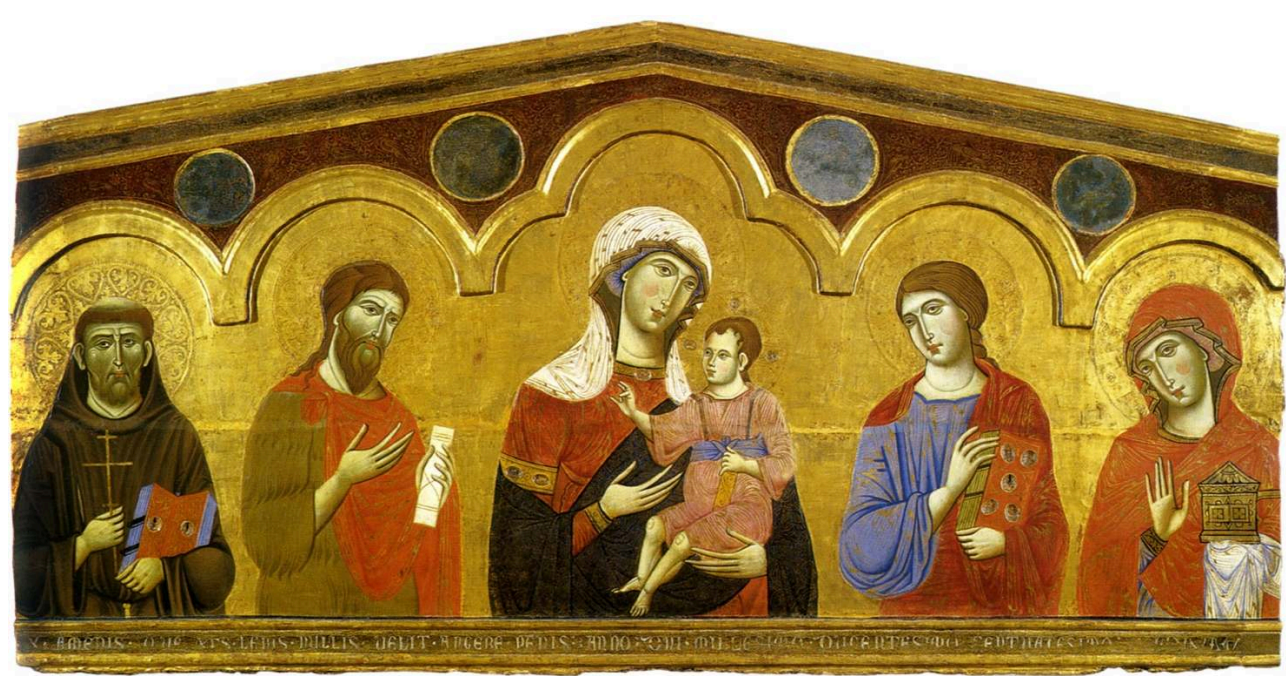

Guido da Siena, Dossal Nº7 (Vierge à l'Enfant avec Saints), v. 1270, tempera sur bois, 96×186 cm, Pinacoteca nazionale, Sienne

cliché Sailko, Wikimedia Commons

4 Puis, en 1270, Guido da Siena l'associe à François dans le Dossal n 7 (fig. 3), le premier exemple de ce type d'objet qui nous soit parvenu. Par la suite l'évocation par les franciscains de la sainte à Assise même, dans la basilique inférieure, dès les premières années du Trecento, a marqué les esprits pour des générations. Les fidèles qui piétinaient devant l'accès aux reliques de François $^{5}$ ne pouvaient oublier la belle Madeleine extatique aux cheveux dorés, veillant du haut de sa lunette (fig. 4), tandis qu'ils attendaient de parvenir au but de leur pèlerinage. 
Fig. 4

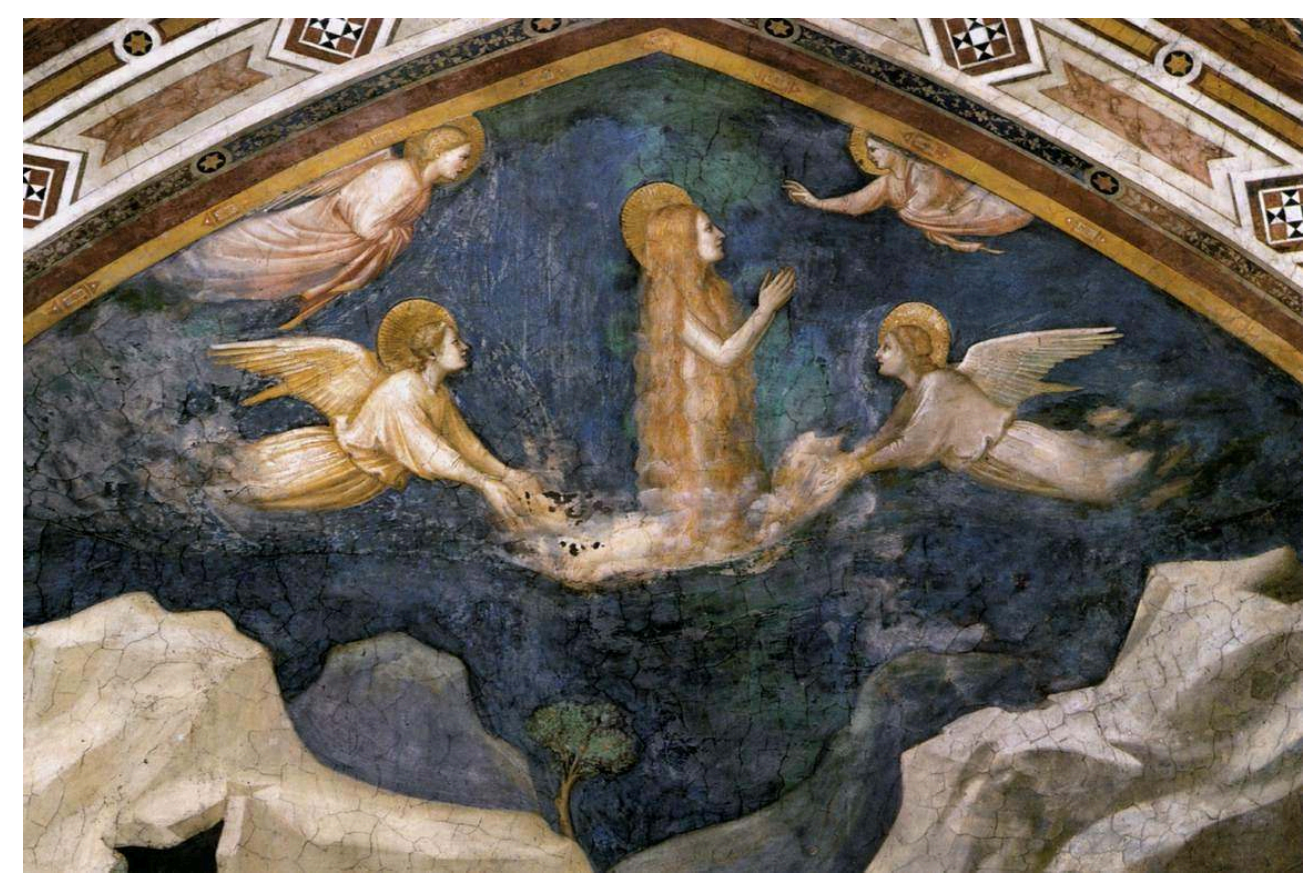

Giotto, S. Marie-Madeleine en extase, v. 1308, fresque, Chapelle de la Madeleine, Basilique Inférieure, Assise

Cliché World Gallery of Art

5 La Madeleine dans la Pietà (Onction) (fig. 5) de Pesaro - elle aussi dans les Marches témoigne d'une autre formulation franciscaine de la sainte figurée en train d'oindre le corps du Christ mort, et ceci vers 1476, c'est-à-dire en plein dans les années de notre enquête. 
Fig. 5

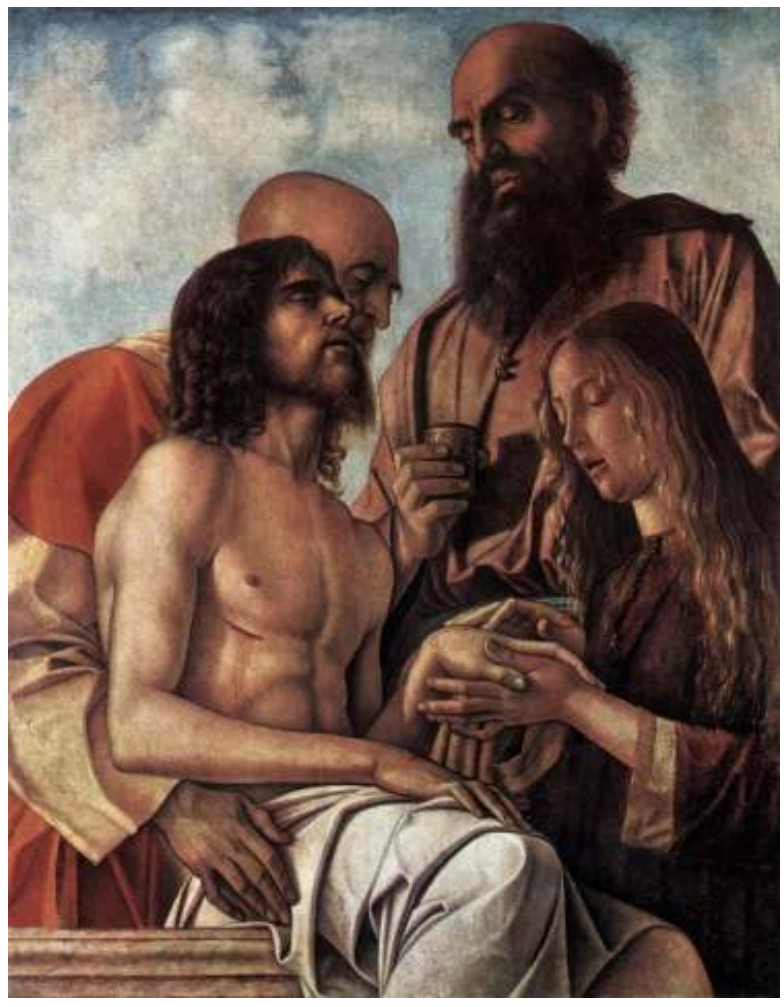

Giovanni Bellini, Pietà (Onction), v. 1476, tempera et huile sur bois, 107 x 84 cm, Pinacoteca Vaticana, Rome

cliché Sailko, Wikimedia Commons

\section{La Madeleine de Montefiore}

6 Le polyptyque dont cette Madeleine faisait partie est aujourd'hui démembré. Une reconstitution récente par Thomas Golsenne suppose une Vierge à l'Enfant au milieu flanquée par Catherine d'Alexandrie, Pierre à gauche, puis par François et la Madeleine à droite. Sur un registre supérieur, chaque saint est couronné par une figure en buste. Un franciscain qui pourrait être Duns $\operatorname{Scot}^{6} \operatorname{couronne}^{2}$ atherine ; Claire d'Assise, Pierre ; une Pietà, la Vierge, un panneau perdu, François; et Louis de Toulouse, MarieMadeleine (fig. 6). 
Fig. 6

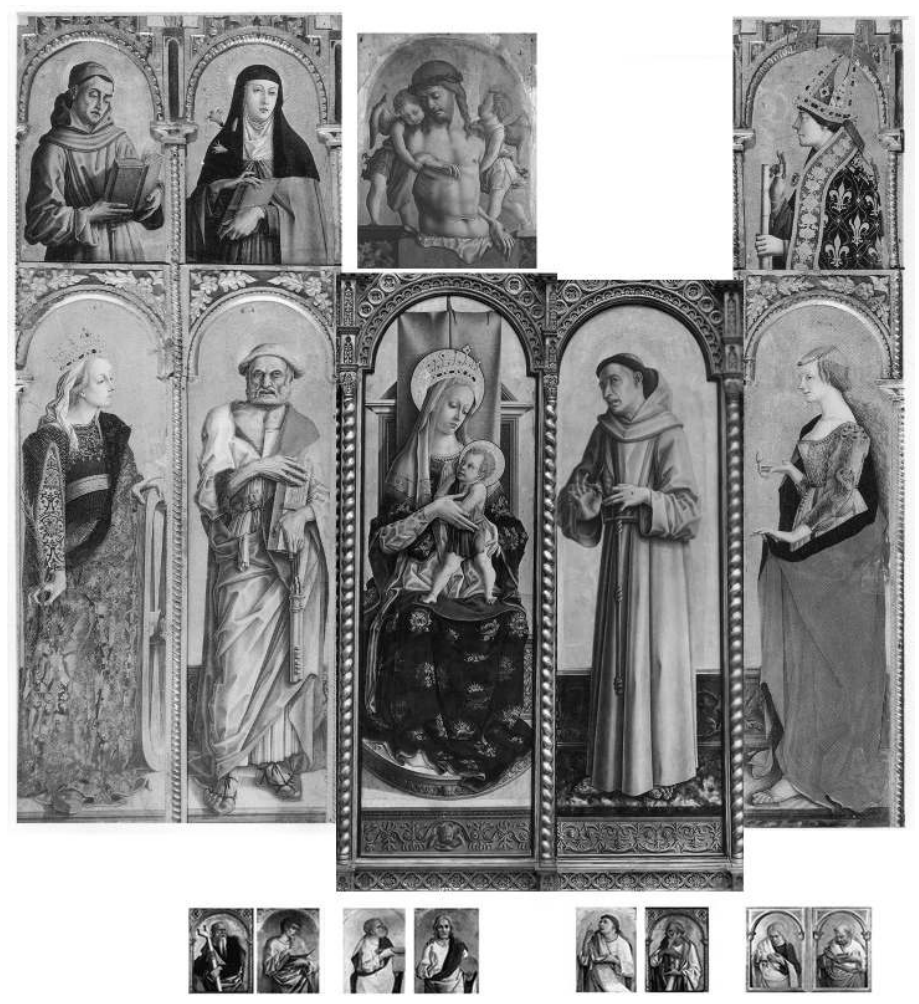

Reconstitution du Polyptyque de Montefiore dell'Aso, démembré

D’après T. Golsenne, Carlo Crivelli et le matérialisme mystique du Quattrocento

\section{Parure et parfum}

7 Tout à fait à droite, donc, aux côtés de François, sur fond d'or, Madeleine se tient dignement, son manteau rouge et vert à la main, mais est montrée au demeurant immobile en strict profil, selon la formule typique des portraits de jeunes filles à marier, depuis la supposée Ginevra d'Este de Pisanello de 1435-40 jusqu'à la Jeune fille en jaune d'Alesso Baldovinetti de 1465 (fig. 7). 
Fig. 7

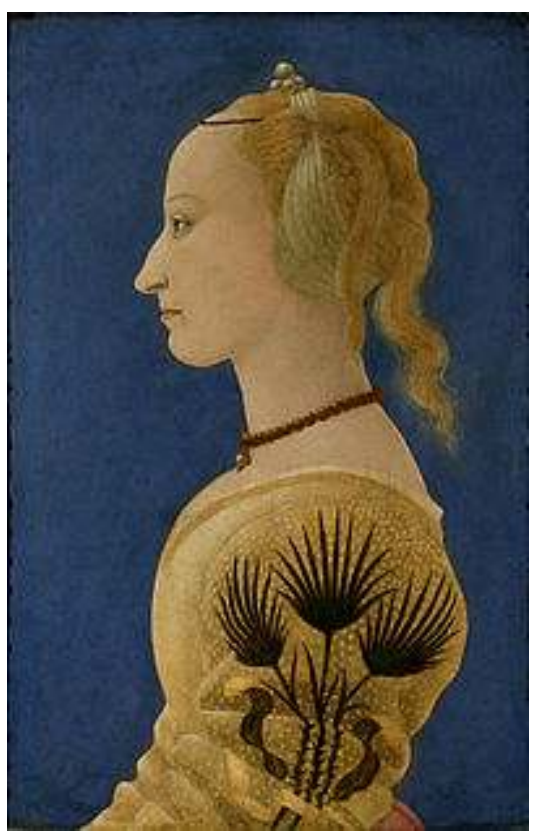

Alesso Baldovinetti, Portrait de jeune fille en jaune, 1465, tempera sur bois, $62,9 \times 40,6 \mathrm{~cm}$. National Gallery, Londres

cliché Erwin Jurschitza, Wikimedia Commons

8 Comme dans ce dernier exemple, sa position met en valeur sa manche en broderies élaborées, le long bras et les doigts délicats. Ces doigts longilignes, comme le corps de la sainte, tiennent son manteau de telle façon qu'elle montre un pied délicat chaussé d'une sandale. Son corsage ouvragé d'or est par contraste bien luxueux et met en valeur la taille fine et les seins sous sa camicia transparente. Sa paume supporte, plus qu'elle ne le tient, son vase de parfum. Vase étrangement représenté car il n'est pas peint mais au contraire simplement ciselé, encore de profil, à même le fond d'or. Le hiératisme de la figure est contredit par le regard en coin de la sainte qui happe le nôtre tandis que ses cheveux châtains semblent désordonnés par une brise ${ }^{7}$. Ce détail l'éloigne de l'apparence des jeunes filles modèles que je viens de citer, dont la coiffure élaborée relevait les cheveux au-dessus de la nuque. Les cheveux magdaléens, le long du dos, sont tenus en une seule queue de cheval par un simple ruban qui semble pouvoir se défaire à tout moment. Sa voilette minimale est toute aussi transparente que la camicia, tenue en place par une couronne de perles avec un rubis au front. La science iconographique pourrait répertorier des symboles tout faits et des sources bibliques plus ou moins explicites : par exemple, le verset bien connu de Proverbes 31, 10, qui déclare la valeur d'une femme vertueuse : mulierem fortem quis inveniet procul et de ultimis finibus pretium eius ("Qui peut trouver une femme forte? Elle a bien plus de prix que ce qui vient des extrémités ", qui est traduit tantôt par rubis, tantôt par perles). Ces bijoux couronnent une chevelure qui est, selon Paul dans I Cor, 11, 15 une gloire autant qu'un voile : mulier vero si comam nutriat gloria est illi quoniam capilli pro velamine ei dati sunt ( " tandis que si une femme entretient sa chevelure, c'est une gloire pour elle parce que les cheveux lui ont été donnés en guise de voile »). Son regard pourrait encore rappeler les paroles de Salomon dans le Cantique des Cantiques 4,9, sur l'Épouse, dont elle est une incarnation: Vulnerasti cor meum, soror mea, sponsa; vulnerasti cor meum in uno oculorum tuorum ( "Tu m’as blessé le cœur, ma sœur fiancée, tu m’as ravi le cœur par un 
seul de tes regards »). Ronald Lightbown a repéré, de la même façon, le sens du phénix sur sa manche qui renaît comme le Christ ${ }^{8}$, résurrection dont la Madeleine fut le témoin privilégié. Cet oiseau serait présenté ici, petite image dans l'image ${ }^{9}$, selon la coutume de représenter une armoirie sur une manche amovible que l'on retrouve dans le portrait en profil de la jeune femme par Baldovinetti (fig. 7).

9 Lightbown est par contre moins sensible à la piste qui m'intéresse plus, un ressort qui sera repensé par la suite dans la Madeleine de Carpegna (fig. 2) : si le phénix renait d'un feu, Marie-Madeleine, lors de sa conversion, est embrasée d'amour. Grégoire le Grand écrit d'elle : «[...] l'âme de l'homme qui ne cherche pas la vision de son Créateur est tristement dure, parce qu'en elle-même elle reste froide. Mais si elle commence à brûler du désir de poursuivre celui qu'elle aime, elle se fond au feu de l'amour [...]. L'âme est abattue, la lumière même lui pèse, et dans ce feu, elle est décapée de la rouille de ses péchés, et le cœur embrasé, tel de l'or terni à l'usage, retrouve son bel éclat à travers le brasier. $\gg^{10}$ Le phénix, comme Lightbown l'a remarqué, est un oiseau qui renaît d'un nid enflammé d'épices ${ }^{11}$. En fait, il s'agissait, selon Hérodote, de la myrrhe ${ }^{12}$, un des parfums de la Madeleine, selon le franciscain Ubertin de Casale ${ }^{13}$, un parfum en goutte, qui pleure comme la dolente myrrhophore au tombeau du Christ. C'est l'odeur qui parfume les seins de l'Épouse ${ }^{14}$. Il s'agit donc de la Madeleine elle-même. Le symbole renforce son identité christique ${ }^{15}$. Elle est depuis le Moyen Âge dénommée l'amica, la dilectrix de l'homme-dieu ${ }^{16}$, voire sa Sponsa selon le Cantique des Cantiques ${ }^{17}$. En effet, ces manches figuraient avec d'autres cadeaux vestimentaires, dans les trousseaux des jeunes épouses, offertes par leur promis, par exemple ${ }^{18}$. Les manches figuraient dans les romans courtois d'un autre siècle, où les chevaliers portaient celle de la demoiselle aimée comme un gage, en son honneur ${ }^{19}$. Thomas Golsenne a très bien démontré que c'est précisément pour un milieu bercé de ce genre d'usages courtois et fastueux que peint Crivelli ${ }^{20}$. À Venise, on faisait sans doute parfumer ce type de manche ${ }^{21}$. Mais ce n'est pas tout. En fait, tout se passe comme si l'image mise en abîme sur la manche d'un côté figurait le parfum impossible à rendre, qui se diffuserait depuis le récipient tenu par l'autre main. Il faut regarder tout d'abord de près ce vase si particulier (fig. 1b). 
Fig. 1b

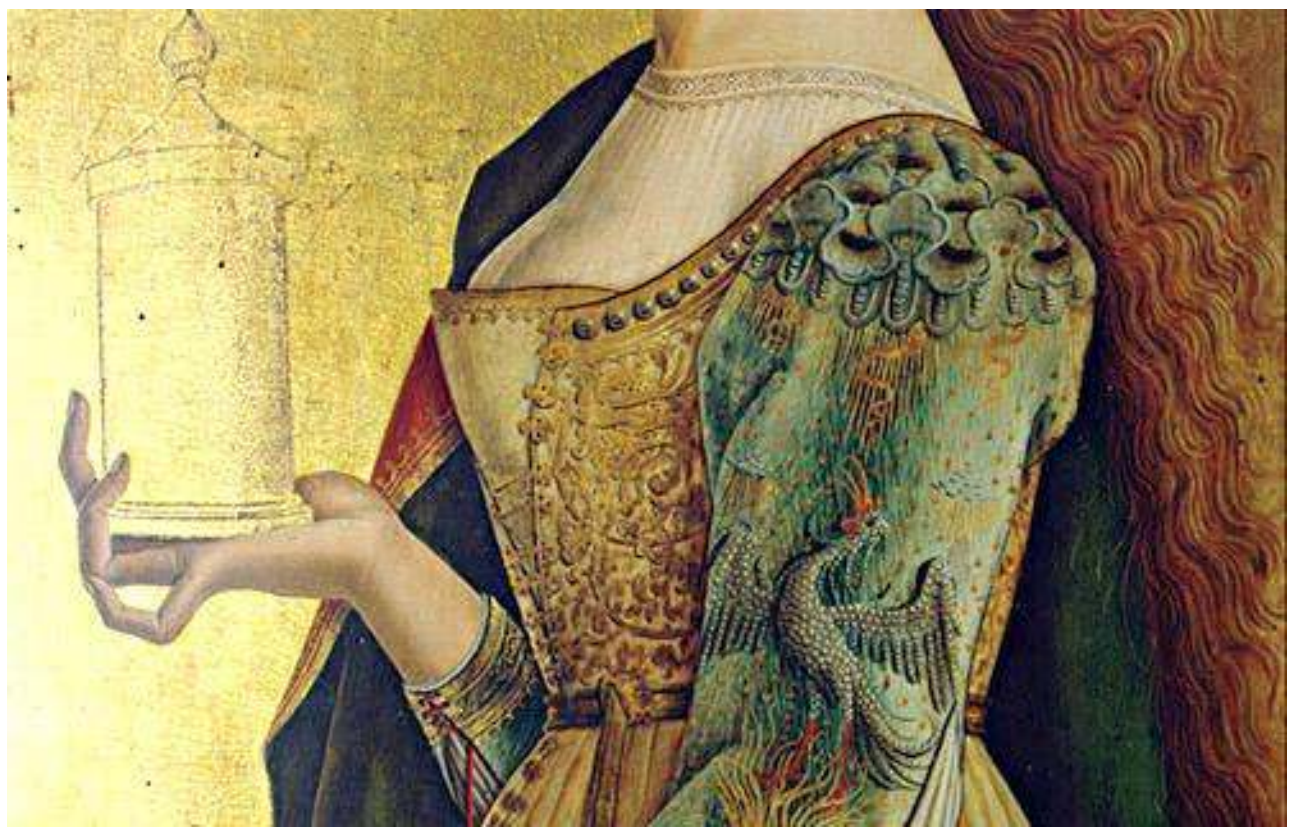

Carlo Crivelli, S. Marie-Madeleine, montefiore dell'Aso (détail)

Les évangiles synoptiques parlent d'un vase d'albâtre ${ }^{22}$. Pourtant, le fait de représenter l'attribut le plus insigne de Marie-Madeleine comme un objet fait en or connaît une longue tradition qui remonte au moins au dossal de Guido da Siena en 1270 (fig. 3), principalement en milieu franciscain. Ciseler le vase dans la feuille d'or du fond n'est pas non plus sans précédent. Crivelli aurait pu voir un exemple à Valleromita peint par Gentile da Fabriano (fig. 8), même si celui-ci est bien plus orné en pinacles délicats, à la façon d'un reliquaire. 
Fig. 8

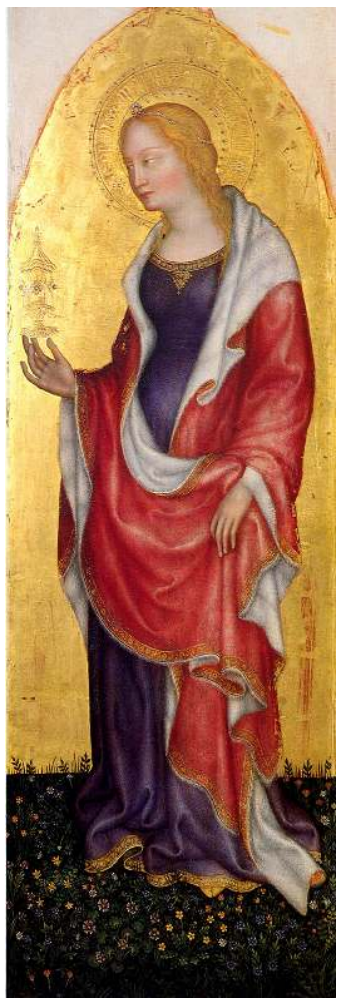

Gentile da Fabriano, Marie-Madeleine, détail du Polyptyque de Valleromita, v. 1405-1410, tempera sur bois, $117 \times 40 \mathrm{~cm}$, Pinacoteca di Brera, Milan

$\mathrm{DR}$

Un peu plus tard, Michele di Matteo cisèle lui aussi de nouveau un vase tenu par la Madeleine dans le Polyptyque de sainte Hélène (fig. 9).

Fig. 9

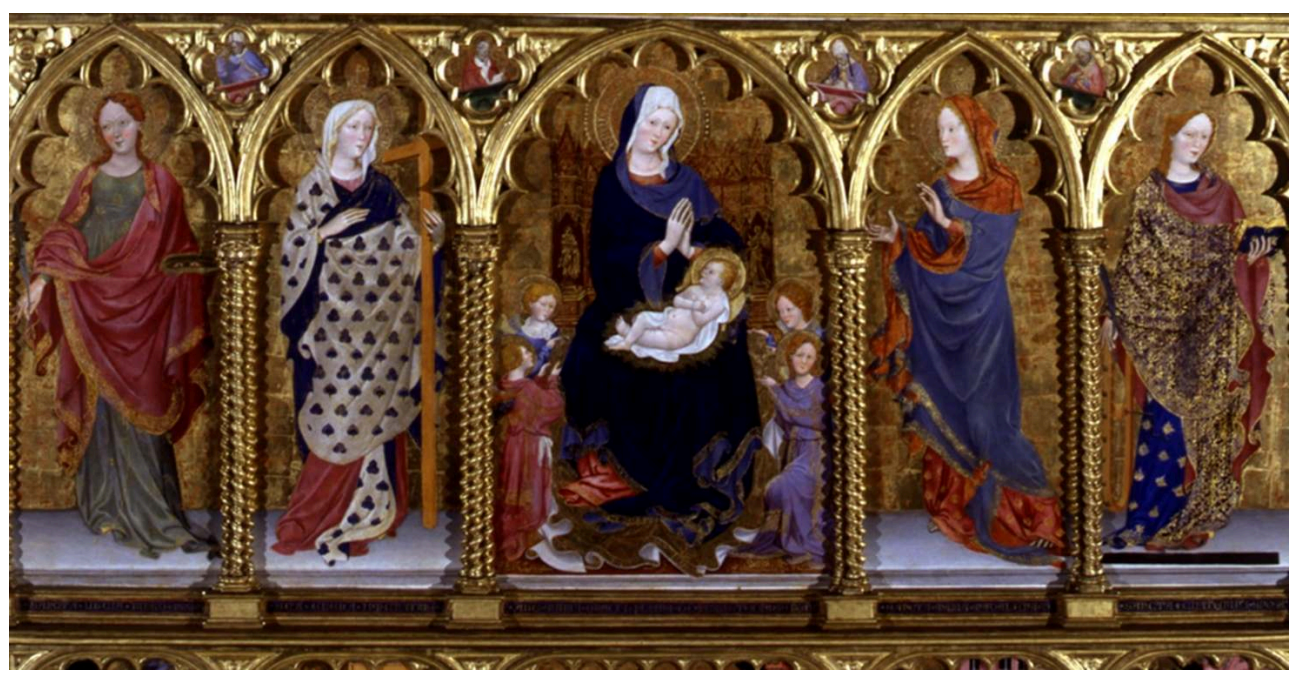

Michele di Matteo, détail du Polyptyque de sainte Hélène, après 1427, tempera sur bois, Galleria de l'Accademia, Venise

DR 
Cette formule est donc connue dans le milieu des frères mineurs et dans le contexte vénéto-marchisan. Thomas Golsenne a parfaitement montré comment les fonds d'or fonctionnent dans l'œuvre de Crivelli, dans la lignée des icônes byzantines où l'or figure la présence divine, au point que l'on pouvait s'y référer comme à la « lumière ». Il est clair que c'est le cas ici, étant donné que Crivelli utilise la même technique pour figurer le nimbe des saints partout dans ce retable, celui de la Madeleine incluse ${ }^{23}$. Dans le Sermo attribué à Odon de Cluny, le plus répandu sur Marie-Madeleine, figure un mot qui revient comme un leitmotiv : fundere, c'est-à-dire, se diffuser, mot qui s'applique et à la lumière et au parfum ${ }^{24}$. Suite à l'analyse qu'en fait Dominique Iogna-Prat, j'ai démontré l'existence d'une tradition, dans des mouvances franciscanisantes, d'une Madeleine au vase doré, dont les rayons peuvent aussi être une façon de figurer le parfum qui s'en dégage, qui a selon l'Évangile, rempli la maison de son odeur ${ }^{25}$. Par glissement, selon la Légende Dorée, la sainte prédicatrice avait une parole parfumée, dont la fragrance convertit ses audiences païennes ${ }^{26}$. Elle est ici en effet jumelée avec Catherine d'Alexandrie, autre femme qui prêche avec une éloquence telle qu'elle fait convertir les gentils au christianisme, au culte du vrai Dieu - qui est lumière. De la même façon, la "seconde Madeleine ", Marguerite de Cortone, est décrite comme "un véritable vase de sainteté " abondant en lumière et parfum ${ }^{27}$. Le compagnon de Madeleine dans le retable, immédiatement à sa gauche, est François lui-même qui montre sa plaie au côté qui saigne. Le référent en est bien sûr le sang du Christ dont François est la nouvelle incarnation. Ce sang serait donc, comme le Sang christique, un sang parfuméres un sang qui attire à Dieu. Le manteau rouge de la Madeleine apparaît comme un vestige de ses robes rouges du siècle précédent, semblant toutes teintes en sang christique, comme si elles s'écoulaient des plaies sacrées ${ }^{29}$. On peut discerner une longue tradition plastique de cette pensée figurative quand on regarde comment vase et plaie sont associés en remontant aussi loin que 1270 où Guido da Siena fait rimer cette plaie franciscaine avec le vase doré (fig. 3). Pour montrer que cette réflexion recouvre les aires temporelles et géographiques qui nous concernent, on peut se référer aux parallèles structuraux qu'en fait un autre Vénitien dans les Marches franciscaines, Giovanni Bellini, vers 1476 (fig. 5).

La simplicité du vase peint par Crivelli contraste avec les vêtements précieusement travaillés, voire orfévrés, de la sainte. Si la figure du phénix est une façon de reprendre le feu d'amour qui embrase la Madeleine, ces flammes la purifient et transforment ces scories en or. C'est une façon de comprendre ce corsage ouvragé de broderies en métal doré. Crivelli simplifie le profil du vase par rapport à ce qu'il a dû voir dans la peinture de Gentile da Fabriano à Valleromita (fig. 8), où le vase prend, celui-là, la vraie forme d'un reliquaire. Tout se passe comme si le travail fin qui serait approprié pour un reliquaire du parfum de la Madeleine ${ }^{30}$ se glissait du vase vers les tissus et ornements qui parent son corps. Et Madeleine est bien un vase - vase de sainteté comme tous les élus mais surtout en tant que femme et femme identifiée d'abord par le vase qu'elle porte. Un hymne chante « De bassin devenue coupe / Passée de la fange à la lumière / Transposée en vase de gloire $»^{31}$. C'est comme si elle devenait sa propre fiole de parfum, ou pour reprendre les termes de Thomas Golsenne, elle devenait son propre reliquaire ${ }^{32}$. Le phénix communique ainsi la fragrance de myrrhe, qui, comme des seins de la Sponsa, s'en échapperait. Crivelli utilise régulièrement les habits précieux pour transcrire la sainteté, la beauté intérieure et extérieure des élus de la cour céleste, et leur noblesse spirituelle. Ici les robes splendides de Marie-Madeleine et Catherine 
d'Alexandrie se mettent mutuellement en valeur : la modestie de l'une (la hauteur du col est digne d'une jeune fille sage comme celle de Baldovinetti, fig. 7) accentue la transparence au niveau des seins et le décolleté de l'autre. Ensemble, les deux parures mettent en valeur le dénuement des figures masculines de Pierre et de François. Une dialectique de l'habit fastueux versus l'habit ascétique est déjà mise en place par Guido da Siena deux siècles plus tôt quand il oppose la bure de François et le cilice de JeanBaptiste aux stries flamboyantes de rouge et d'or de la Madeleine et de Jean l'Évangéliste (fig. 3). Ici le contraste se joue entre les saints masculins austères au centre et les saintes féminines à l'extérieur, habillées de toilettes fastueuses.

Quelques détails viennent brouiller pourtant cette symétrie entre les deux saintes, notamment le pied de la myrrhophore qui s'aperçoit depuis l'ourlet rouge et or de son manteau. Celui-ci n'est pourtant pas habillé d'un escarpin pointu d'une finesse toute curiale, mais plutôt de la sandale que portent les frères mineurs en signe de leur vœu de pauvreté. Or la sainte courtisane est surmontée dans le polyptyque par Louis de Toulouse, ce prince de famille angevine (dynastie qui était placée sous la protection particulière de Marie-Madeleine ${ }^{33}$ ) qui a refusé la couronne de Naples pour embrasser une vie de frère rigoriste jusqu'à ce qu'il soit frustré dans son désir de pauvreté absolue en étant nommé évêque.

Fig. 10

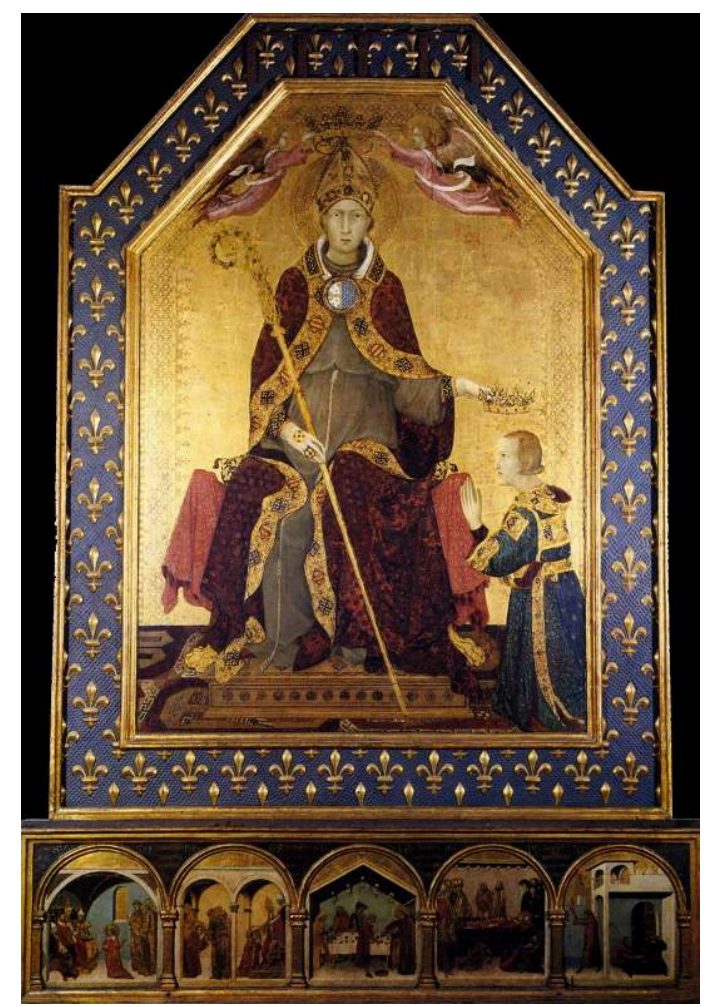

Simone Martini, Saint Louis de Toulouse, v. 1317, tempera sur bois, $200 \times 138$ cm, Museo di Capodimonte, Naples

cliché Erwin Jurschitza, Wikimedia Commons

Crivelli suit l'iconographie traditionnelle mise en place depuis Simone Martini, contemporain de la canonisation du jeune ascète vers 1317 (fig. 10), c'est-à-dire en le figurant avec son manteau d'évêque qui recouvre une bure franciscaine. L'opposition 
entre magnificence d'un côté (l'éclat des atours de la Madeleine) et dénuement (la sandale de Dame Pauvreté) de l'autre est claire. Quant à Louis de Toulouse, les codes de sa représentation sont bien ancrés depuis le Trecento, mais si Carlo Crivelli avait pu voir le panneau de Martini, ou bien n'importe quelle autre de ses œuvres, il en aurait sûrement été ravi. Le Siennois - avec son travail minutieux d'orfèvrerie, de reliefs en gesso, de poinçonnage, de vêtements somptueux, ajoutés à un luxe tout courtois et une ligne nerveuse et gothique - aurait sans aucun doute eu de quoi plaire au Vénitien. Des routes relient Ascoli Piceno, où il était installé dans la Marche d'Ancône, à Sienne, à Naples ou encore à Assise où l'on retrouve un ensemble de figures bien similaires de la main de Simone à l'entrée de la Chapelle Saint-Martin dans la basilique inférieure. Ici aussi se rassemblent, entre autres, Claire d'Assise, Catherine d'Alexandrie, MarieMadeleine et Louis de Toulouse. Un voyage à Assise n'est pas du tout impossible. Visiter les images de la maison-mère de l'ordre pourrait même s'imposer à un peintre si relié à leur patronage $e^{34}$, peut-être avant d'entamer un imposant retable à leur demande. Bien sûr, en tant que haut lieu d'imagerie dans la péninsule et pèlerinage extrêmement populaire, il aurait pu y aller à n'importe quel moment de sa carrière ou même dès sa première jeunesse en tant que pèlerin. Il est une autre raison de faire le lien entre ce qui se voit à Assise de la main de Simone Martini et à Montefiore dell'Aso de celle de Carlo Crivelli. Dans cette église se trouve le tombeau du prélat Gentile da Partino qui a justement supporté les frais de la chapelle Saint-Martin dans la basilique inférieure, et le retable crivellesque serait à la fois une digne continuation de son œuvre de mécène artistique et un tribut à ce cardinal ${ }^{35}$.

\section{Vase et grâce}

On peut voir un souvenir de la chapelle d'Assise dans la façon dont Marie-Madeleine tient son vase (fig. 11). 
Fig. 11

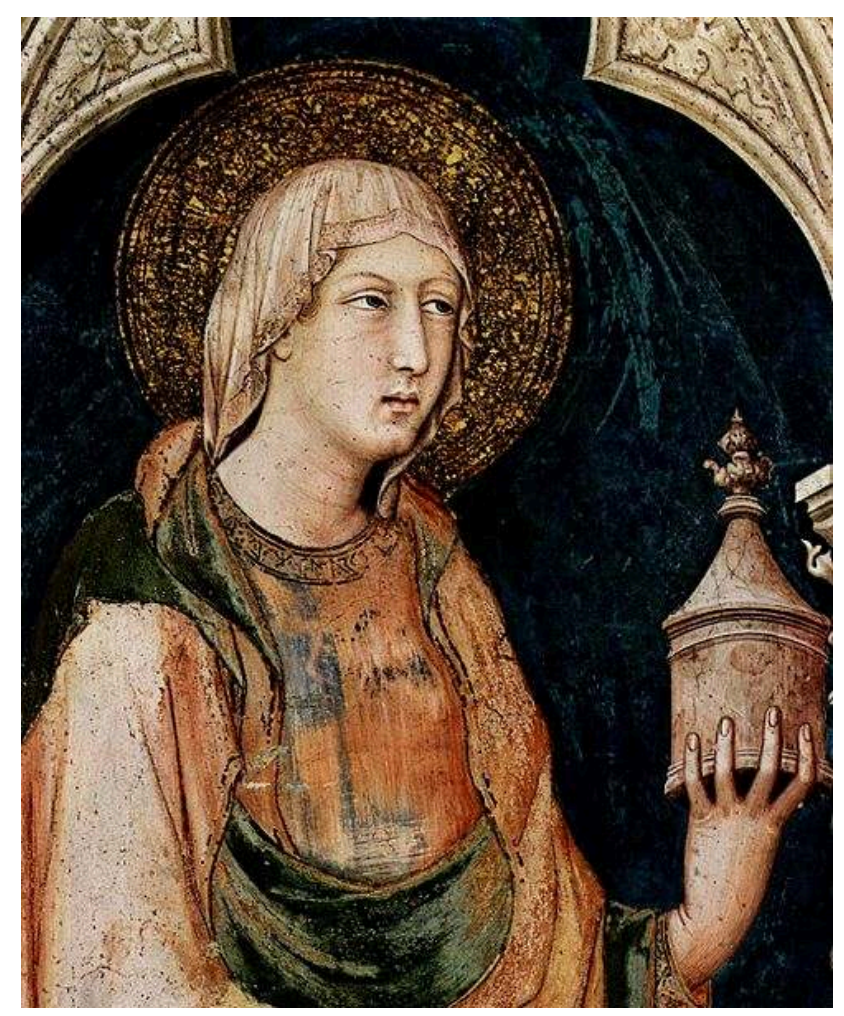

Simone Martini, Marie Madeleine, détail v. 1315-17, fresque, Chapelle de Saint Martin, Basilique Inférieure, Assise

cliché Wikimedia Commons

Chez Simone c'est un geste quelque peu maladroit, une tentative d'in scorcio en quelque sorte. Chez Crivelli les mains maniérées de la Madeleine n'ont aucune gaucherie : elles sont tout d'harmonie avec un vase immatériel. Dans les deux cas, pourtant, il s'agit d'une façon bizarre de placer doigts et mains. En ce sens, l'une pouvait inspirer l'autre. La différence d'interprétation pouvait être due à cette métamorphose des mœurs au cours du Quattrocento analysée par Paul Hills ${ }^{36}$. Les nouvelles règles de civilité voulaient que l'on manipule certains contenants, par exemple les verres de vin, de façon à réprimer toute inélégance, toute balourdise, et à manifester une sprezzatura dans le mouvement corporel. Si le changement est dû à l'avènement du verre, l'épicentre de la production de cette matière (et du parfum par ailleurs ${ }^{37}$ ) est bien la patrie de notre peintre. Comme tout Vénitien il était bien familier avec la délicatesse et la grâce - nous y reviendrons - avec lesquelles il faut manipuler de tels objets. Or ici ce n'est pas un vase de verre à l'image de celui que représente un Antonello de Messine à ses débuts vénitiens (fig. 12) : il est figuré de façon encore plus immatérielle. 
Fig. 12

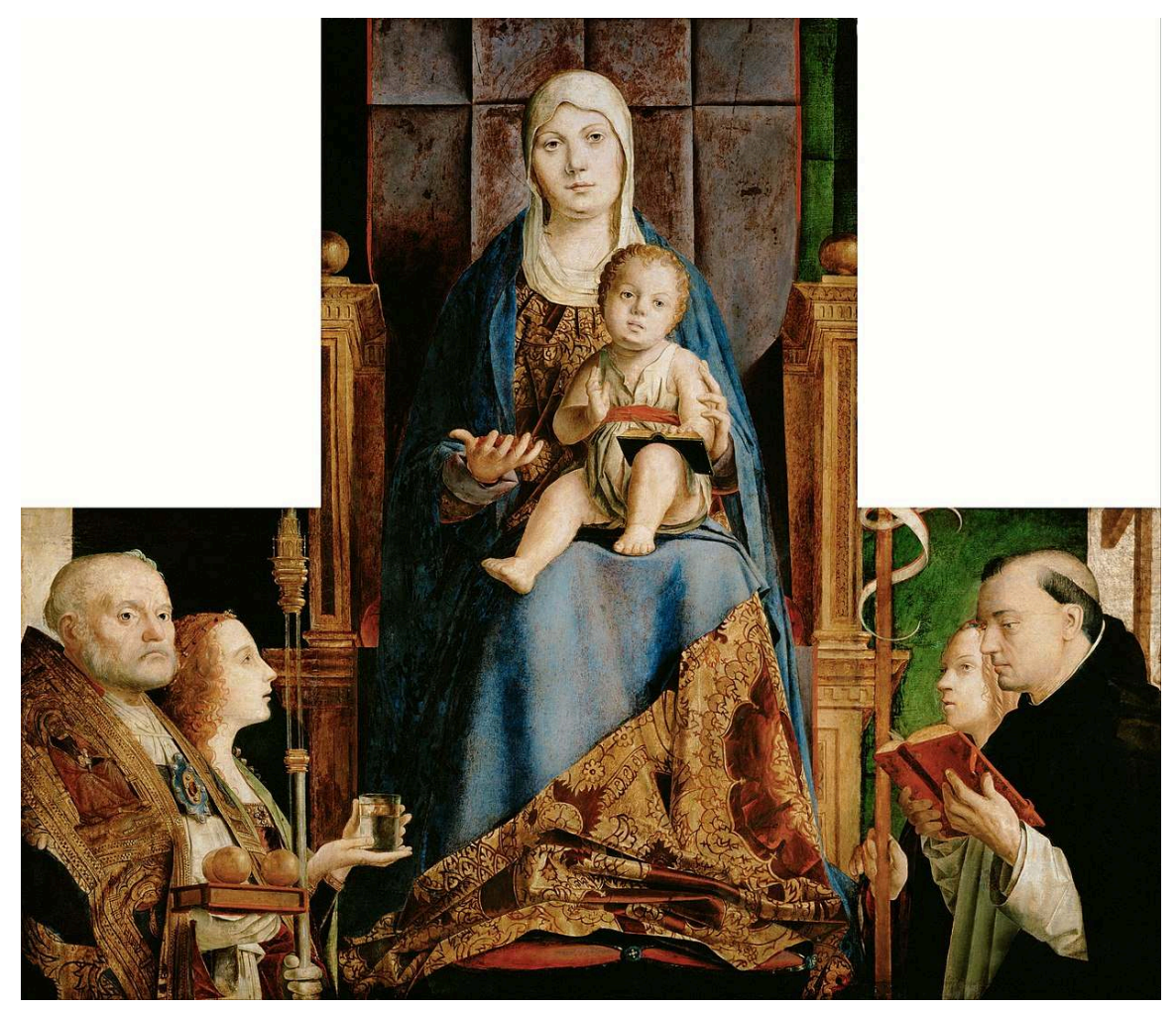

Antonello de Messine, Pala de San Cassiano, 1475-1476, huile sur bois, panneau central : $115 \times 135 \mathrm{~cm}$, panneau de gauche 55,9 × $35 \mathrm{~cm}$, panneau de droite 56,8 × 35,6 cm, Kunsthistorisches Museum, Vienne

Cliché Google Art Project

Il semble sans consistance, parfaitement sans substance et il faut bien une prise " pleine de grâce " pour manier un contenant qui est une espèce d'anti-objet, un nonobjet en apesanteur. Chez la Madeleine crivellesque, la grâce physique va de pair avec la grâce métaphysique, quand on réfléchit aux paroles de Raban Maur qui s'extasie, citant le livre des Proverbes, sur « la grâce de ses lèvres » ou sur ses charmes et de sa beauté plus généralement ${ }^{38}$. Aussi, ayant quitté sa vie de pécheresse, c'est encore la grâce qui la caractérise, non seulement pleine de grâce mais trop-pleine. Il s'agit du passage d'inspiration paulinienne dans la Légende Dorée, souvent appliqué à ce personnage: "Après sa conversion, elle fut magnifique par la surabondance de la grâce, car là où avait abondé le péché, surabonda la grâce $»^{39}$. Cette grâce s'aperçoit encore dans le petit geste du pied de la pécheresse, telle que l'imagine Crivelli. Elle le montre. Geste assez scandaleux qui possède en quelque sorte la même tonalité que la camicia transparente sur ses seins ${ }^{40}$, comme la voilette qui ne cache pas du tout une chevelure abondante prête à se défaire avec ce vent qui la soulève. Le semi-nudité de ce pied que nous montre la courtisane pénitente, dans ce contexte visuel, porte une signification bien différente que celle du pied de la chaste Catherine emmitouflée dans ses habits opaques.

\section{La séduction et la tradition visuelle magdaléenne}

19 Le regard en coin, regard aguicheur vient parachever cet effet de grâce surabondante qui saisit l'œil du spectateur déjà pris par les rets «fractaux » ${ }^{41}$ des broderies de son 
corsage. Ses beaux yeux, selon Bernardin, font partie de ses façons de pécher : « Togli il quarto modo di peccare, cioè cogli occhi. Imperò che ella aveva bellissimi occhi e sempre mai gli balestrava » («Retire la quatrième façon de pécher, à savoir avec les yeux. Car elle avait de fort beaux yeux et jamais elle ne les dardait $)^{42}$ ou bien encore "Degli occhi vani, col balestrare in qua e in là » (« des yeux vaniteux, dardant ça et là »). Dans le mot balestrare il y a certes l'idée des regards "jetés » ici et là, errant donc, mais aussi un regard qui frappe comme une flèche, qui blesse (balestrare vient de balestra, l'arbalète). L'on songe justement à l'œil de la Sponsa qui blesse le cœur de son amant, dans la citation du Cantique que l'on a vue plus haut, Inerasti cor meum, soror mea, sponsa; vulnerasti cor meum in uno oculorum tuorum ( Tu m'as blessé le cœur, ma sœur fiancée, tu m'as ravi le cœur par un seul de tes regards ", Cantique des Cantiques 4,9). C'est un lieu commun des moralistes qui fustigent les demoiselles ne tenant pas leur regard modestement baissé. Ce regard s'intègre dans une dynamique de séduction du spectateur.

Fig. 13

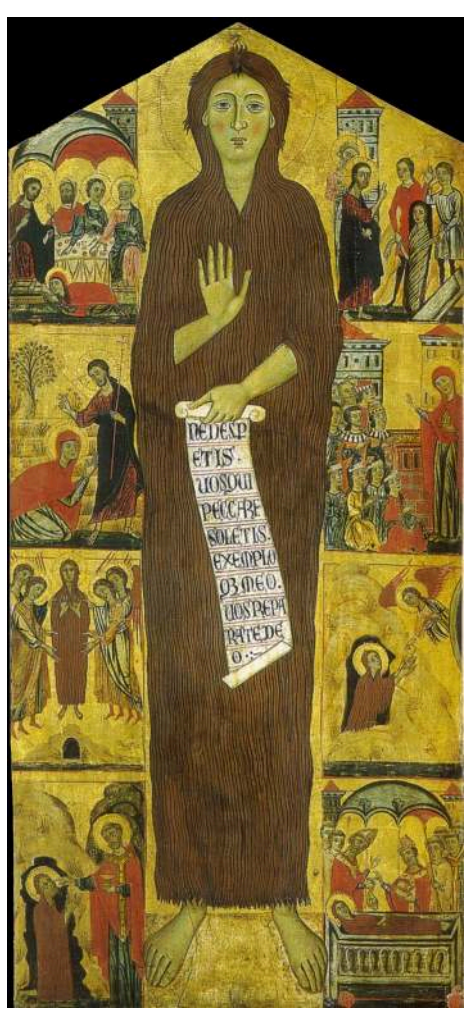

Maître de la Madeleine, Marie Madeleine entourée de huit scènes de sa vie, v. 1285, tempera et or sur bois, $178 \times 90 \mathrm{~cm}$, Galleria de l'Accademia, Florence

cliché Sailko, Wikimedia Commons

C'est une même dynamique que suit l'iconographie magdaléenne dans l'Italie médiévale depuis le panneau florentin du Maitre de la Madeleine de 1285 (fig. 13) et bien développé à Assise où, en toute probabilité, Carlo Crivelli a dû se rendre. Il s'agit d'un éros anagogique qui s'appuie sur la particularité de la sainte peccatrix, transportant le spectateur des beautés matérielles vers les beautés immatérielles comme autant de voiles - ici déjà si transparents - dionysiens ${ }^{43}$ qui s'enlèvent comme dans un effeuillage sacré. 


\section{La Madeleine de Carpegna}

21 Vers 1492 une pensée similaire anime une autre Madeleine en pied (fig. 2). Que s'est-il passé dans la réflexion plastique derrière la représentation de la sainte depuis au moins vingt ans ? La comparaison - et le contraste - sont riches de sens.

\section{Parfum et parure : vingt ans après}

La Madeleine carpegnate est fort proche de la Madeleine montefioraine : un beau corps dans des robes recherchées et raffinées, des mouvements élégants, une belle et abondante chevelure animée par le vent. On retrouve ici encore un regard en coin qui attrape le spectateur. Comme la précédente, celle-ci provient d'une église franciscaine. Cette fois-ci les moyens sont en toute probabilité fournis par une dame du grand monde, une comtesse locale, Caterina, originaire de la famille noble des Sardini et qui vouait un culte tout particulier à la courtisane pénitente ${ }^{44}$, qui selon des légendes destinées à une bien longue vie avait le même rang social qu'elle ${ }^{45}$. La différence majeure entre les deux images résulte de cette piété : cette fois il n'y a pas de panneaux conjugués dans un réseau de sens comme dans un retable traditionnel et, de marginale, Madeleine est devenue centrale. La sainte est le seul objet de vénération et doit porter seule le sens dévotionnel de sa pala. C'est peut-être la raison pour laquelle la plupart des motifs et dispositifs y sont comparables, mais plus élaborés, voire plus extrêmes.

Le regard qui, selon l'accusation de Bernardin de Sienne, balestrava, qui darde, est souligné par un trait noir sur la paupière, comme du maquillage ${ }^{46}$. Le front est haut, ses fins sourcils sont épilés au dernier degré et ses cheveux sont passés de châtains à blonds. La teinture est très fréquente pour les dames italiennes d'alors, nonobstant les reproches du prédicateur. Coiffée de façon extravagante, elle porte un diadème qui est, comme la bordure de son auréole, en relief, tissé dans les tresses d'une ligne sinueuse, serpentine même et qui semble se mouvoir à la faveur d'une "brise invisible", warburgienne $e^{47}$. Ses bouclettes n'ont rien de naturelle, en contraste avec celles de Montefiore. Comme pour souligner cet effet d'artificialité, la broderie de la manche fait rimer vrilles métalliques et cheveux ondulés. Il n'y a pas cette fois de phénix qui décore la manche, toujours amovible et prête à s'offrir à un galant. Pourtant le thème du parfum se prolonge, car sur l'épaule apparaît un relief de rose stylisée. Les stries semblent figurer, par la lumière dorée, une nouvelle fois, le parfum qui se dégage du vase. Le peintre continue de représenter l'odeur du contenu à (sa) gauche et le contenant à (sa) droite, comme à Montefiore. Il va pourtant plus loin. Car si l'épaule gauche porte une rose en broderies dorées, fleur que l'artiste représente en léger relief, une rose vive et d'autres fleurs pendent juste au-dessus de son épaule droite et du vase de parfum. Du côté droit du tableau sont accrochées quelques petites fleurs mais qui se sont tournées vers le fond du tableau. La rime visuelle entre les vrilles de la fleur à l'épaule gauche et les boucles que les encadrent, intègre, par extension, la chevelure dans le réseau des signes visualisant les odeurs ${ }^{48}$. D'autant plus que les tresses ondulantes de sa droite font écho à l'anse courbée de son vase de parfum. Sur son corsage il n'y a plus d'arabesques abstraites, mais encore des fleurs exubérantes ${ }^{49}$ qui épousent la forme de ses seins, tels les seins parfumés de la Sponsa. Les boutons qui ne ferment pas le corsage sur la poitrine, et même sa ceinture étaient en pratique souvent parfumés ${ }^{50}$. Même le nez pointu et retourné typique des saintes de Crivelli se trouve ici 
quelque peu exagéré au niveau des narines, comme si la courtisane respirait ces saintes fragrances. Elles lui sont offertes autant qu'elle les offrent, à l'image du va-et-vient entre le spectateur, le fond illusionniste du tableau et sa surface. Les pastiglie a relievo technique qui est partie intégrante du répertoire formel de ce peintre et souvent indiquée comme signe d'une régression artistique - sont présentes ici en abondance, notamment sur le costume de la sainte : les attaches et bordures de sa manche où siège la rose ; les boutons et liseré de son corsage ; la boucle et la décoration de sa ceinture ; son diadème et le rebord de son auréole. Bien loin d'être rétrogrades, ces pastiglie a relievo font, au contraire, partie de la sophistication du tableau et de son fonctionnement cultuel.

Ces protubérances seraient plutôt une façon de représenter une offrande à la présence du saint dans une image de culte. Les guirlandes, comme dans tant de ses œuvres, fonctionneraient de la même façon, comme des dons pour honorer le ou la sainte figuré $e^{51}$. Les reliefs se cantonnent largement ici à la toilette, glissant du cadeau typique (manche, boucle de ceinture, diadème) du client amoureux à une courtisane , ou de fiancé à fiancée, à l'offrande de piété pour une sainte. Dans chaque cas, il s'agirait d'une sorte d'échange entre la figure peinte et son spectateur par le truchement de la surface du tableau, dont la Madeleine semble, par son pied gauche, prête à sortir. Comme dans les icônes qui ont tant influencé la pensée du peintre sur son métier, cette sainte possède une présence réelle, voire active. Ses mèches flottantes se superposent au gesso qui entoure son nimbe : elles ressortent donc du tableau en trompe-l'œil pour donner l'illusion de voltiger dans l'espace du spectateur, effet accentué encore par le diadème, lui aussi en relief. Active, la Madeleine l'est aussi en recherchant l'attention du spectateur avec son regard dirigé vers l'extérieur, en coin, le regard de tentatrice dont parle Bernardin.

Un autre usage de la pastiglia a relievo se trouve dans son vase à parfum (fig. 2b). 
Fig. $2 b$

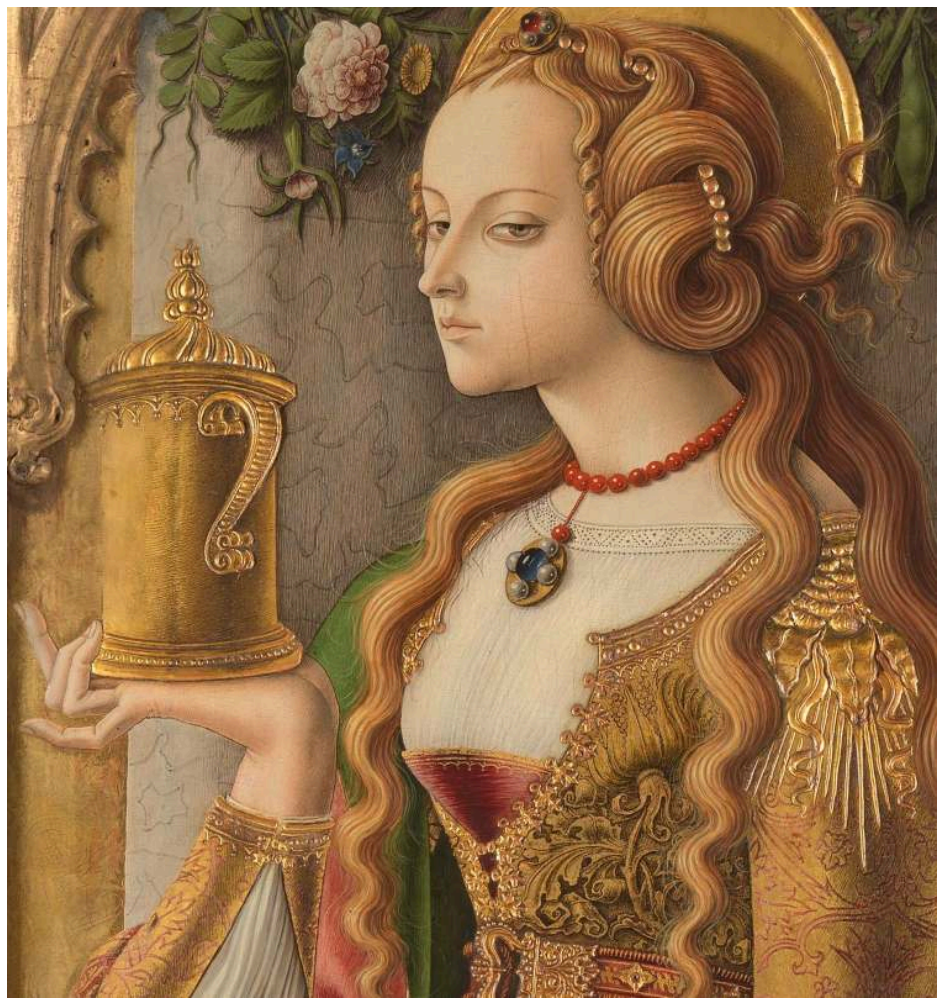

Carlo Crivelli, S. Marie-Madeleine, Rijksmusem, Amsterdam (détail)

Alors que le corps de celui-ci est en feuille d'or, comme le fond (entre plusieurs strates $^{52}$ ), couvercle et anse sont en gesso moulé. Il s'agit ici d'une des différences fondamentales avec le retable de Montefiore. Le vase de la Madeleine de Montefiore s'introduit dans le fond d'or, dans la «lumière » sacrée, tout comme son nimbe. À Carpegna, ces dorures se projettent dans l'espace du dévot. Crivelli a utilisé le relief pour les anses, à l'endroit où le vase peut être manié. Je ne connais pas d'autre image $\mathrm{du}$ vase de la Madeleine en Italie avec une anse, d'autant moins une anse en relief ${ }^{53}$. D'ailleurs, la sainte n'utilise pas l'anse pour manier le vase, mais au contraire, elle semble l'offrir au spectateur qui peut alors le saisir par ce moyen. À Assise, le vase de la Madeleine de Simone Martini, lui aussi, se tend vers le spectateur en dehors de son encadrement de colonnettes, par le biais de cette prise in scorcio (fig. 11). 


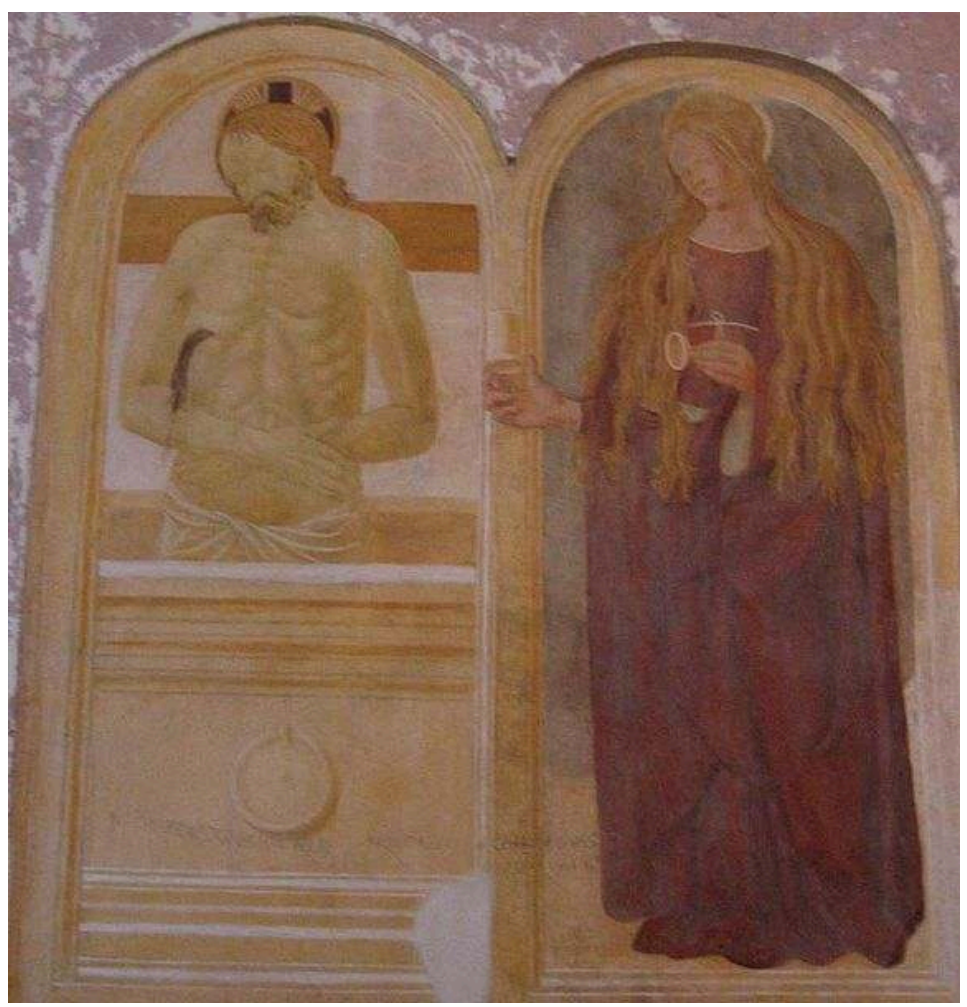

Maître de la Madone di Alvito, S. Marie-Madeleine et Imago Pietatis, fresque, chiesa della Madonna della Sanità, Alatri, Colleprata

Cliché Threecharlie, Wikimedia Commons

Plus proche dans le temps de notre œuvre, vers 1460, le Maître de la Madone d'Alvito (fig. 14) donne à voir, avec un illusionnisme imparfaitement maîtrisé, une Madeleine qui tend le vase vers le Christ dans le compartiment voisin, traversant ainsi l'espace du spectateur. Quelques trente ans plus tard, Crivelli, en représentant un couvercle et une anse tridimensionnels, concrets, offre par illusion tactile la possibilité de saisir et d'ouvrir au moins virtuellement le vase par le ou la dévôtee devant l'autel.

J'ai pu démontrer ailleurs ${ }^{54}$ combien dans l'orbite vénéto-marchisane les matériaux utilisés par les artistes pour représenter le vase de la Madeleine pouvaient aussi fonctionner comme des espèces d'ex-voto. Dans la sacra conversazione de la Pala San Cassiano peinte entièrement à l'huile (fig. 12) par exemple, Antonello de Messine utilise le vase comme un trait virtuose, associant la transparence du verre (cristallo de Murano) à la transparence de l'huile qu'il contient. Or l'huile figure le parfum que la Madeleine offre alors à la Vierge aussi bien que l'huile-médium qu'Antonello offre à son tour par le biais de son métier. Dans une réponse à cette huile votive, Bellini, dans les Marches, à Pesaro (fig. 5), peint une Pietà, une Onction, ajoutant de l'huile à sa tempera. À Pesaro, ce nouveau médium permet au peintre vénitien de faire une sorte d'image miraculeuse qui saigne, à partir de la plaie du côté. Comme sa Pietà de Brera (fig. 15) "qui pourrait pleurer", selon la signature sur le cartellino, il s'agirait d'un panneau doté de présence, d'une image de culte. Comme avec les pastiglie a rilievo de Crivelli, l'huile de Bellini se projette hors de la surface, laisse des traits en épaisseur, comme si le liquide visqueux suintait vraiment de la chair christique, du panneau de bois qui était 
le support de cette apparition, dans un échange sacramentel avec le spectateur qui s'en trouve, à son tour, oint.

Fig. 15

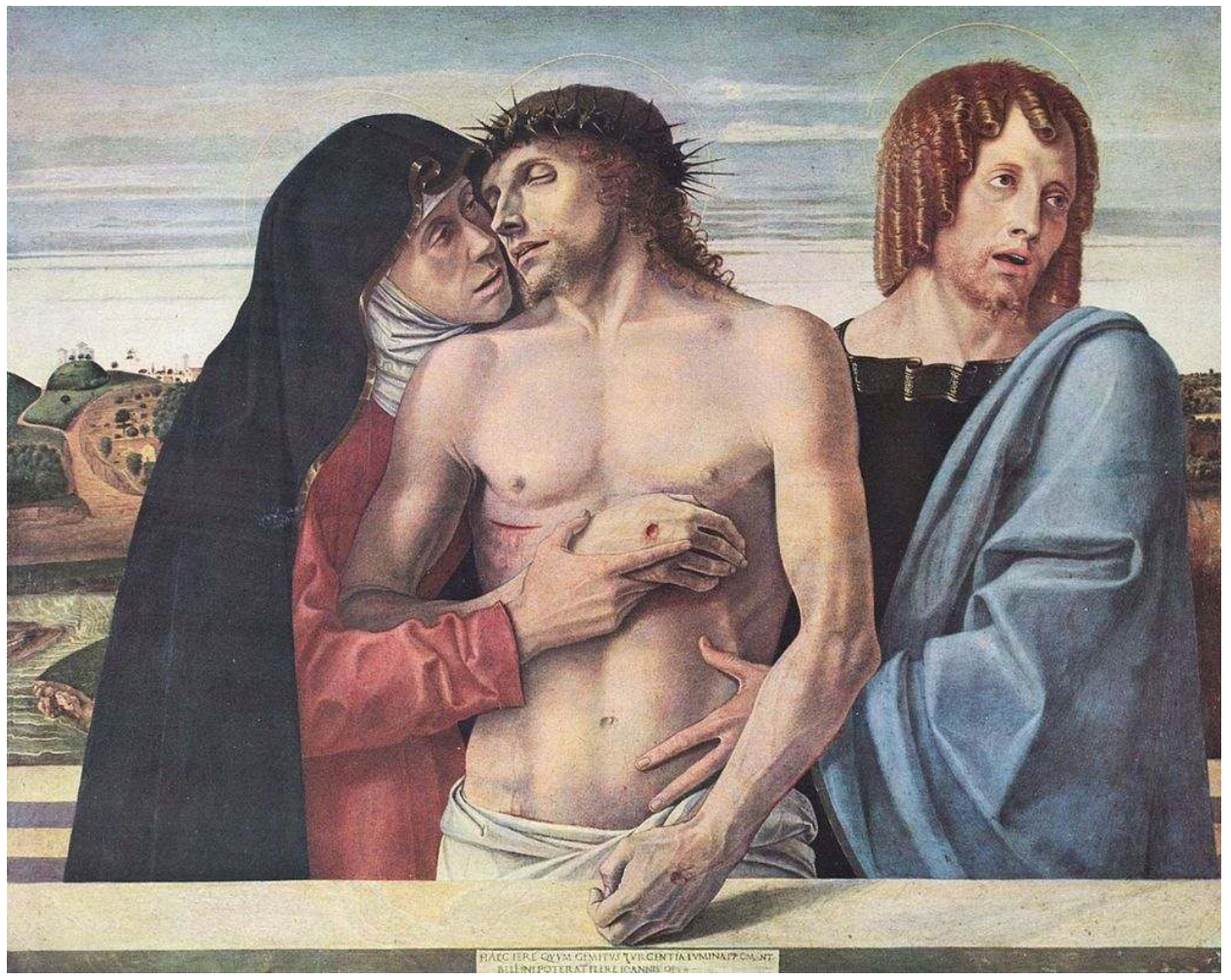

Giovanni Bellini, Pietà, v. 1470, tempera sur bois, 86 x 107 cm, Pinacoteca di Brera, Milan

Cliché Eloquence, Wikimedia Commons

Tout ceci s'insère parfaitement dans une longue tradition vénitienne d'un côté, et de l'autre, dans une tradition franciscaine encore plus ancienne ${ }^{55}$. Cette double tradition tend à faire glisser l'haptique vers l'optique. Comme le dit Thomas Golsenne ailleurs par rapport à la ligne crivellesque : « [...] une sensibilité tactile, la conversion de l'œil en doigt. Il n'est d'ailleurs pas étonnant que le faire du peintre relève lui-même de ce régime tactile. En effet à bien y regarder, en se collant quasiment contre la surface du tableau, quand l'œil se met à avoir des sensations tactiles, c'est tout un système de hachures très fines qui apparait, une sorte de moirage imperceptible qui recouvre toute la surface peinte. ${ }^{56}$. Le toucher est un thème d'autant plus pertinent pour MarieMadeleine qu'elle est souvent décrite ou représentée touchant le Christ, à partir du moment de sa conversion quand elle l'oint. Ensuite, le moment fort de son histoire est précisément l'interdit de ce contact physique après la résurrection lors du Noli me tangere. Ces deux tableaux de Crivelli tournent autour du toucher d'une façon comparable. Dans le premier, personne sauf la Madeleine ne peut entrer en contact avec ce vase sans substance, insaisissable, sauf par les yeux, au spectateur. On songe à la dynamique derrière le Noli me tangere quand la sainte est censée ne témoigner que visuellement de la résurrection de ce corps tant aimé qu'elle ne peut plus toucher. Dans le polyptyque, Crivelli lui rend le privilège de sa première intimité avec le Christ, en transférant la fonction de témoin oculaire chez le dévot devant l'autel. Dans le deuxième tableau, à Carpegna, est proposée une invitation plastiquement explicite à 
s'emparer (du moins par une pensée dévote) du vase, à soulever le couvercle et ainsi humer le saint parfum de Marie-Madeleine.

\section{Pavane : la Madeleine terpsichoréenne}

30 Le binôme haptique et optique entre dans cet échange avec la Madeleine par un autre moyen. Regardons encore ce pied qui pointe en dehors du cadre peint. Avec la position de l'autre pied, la Madeleine esquisse comme une position chorégraphique. Le genou de la même jambe est fléchi sous le manteau. Tout son corps vu de trois-quarts, à la grande différence du profile hiératique de la Montefioraine, semble se contorsionner comme un ressort. Les ondulations de ses boucles en entrelacs viennent souligner cette torsion $^{57}$. On le sait, la grâce fut une marque de sa jeune beauté ${ }^{58}$. Cette qualité apparaît ici de façon plus élaborée que chez la Madeleine de 1470-73 précisément à cause de ce sens de mouvement. Même sa façon de tenir son vase est encore plus maniérée que dans le panneau de Montefiore. Le mouvement l'envahit et elle semble prête à sortir de son cadre, comme ses accessoires le font déjà, et à entrer dans la danse. La danse dans la peinture $d u \mathrm{xv}^{\mathrm{e}}$ siècle italien a notamment été abordée par Michael Baxandall en premier ${ }^{59}$, puis par des travaux plus récents de, par exemple, Olivia Powell et Sharon Fermor et plus généralement dans la culture du Quattrocento par Jennifer Nevile ${ }^{60}$. À ce sujet, il est de mise de se concentrer sur le milieu florentin avec Alberti comme point de repère. Bien que ce dernier s'impose pour la théorie de la peinture quattrocentesque, sa pensée classicisante peut être parfois difficilement applicable au Vénitien et ses valeurs visuelles tardo-gothiques. ${ }^{61}$ Quand l'humaniste se réfère à la peinture par exemple c'est largement dans le contexte de ses recommandations quant à l'istoria ${ }^{62}$. Or ici aucune narration ne vient contextualiser cette unique figure en pied. Seule, elle n'est pas campée dans une composition groupée. Une des prescriptions albertiennes est pourtant bien pertinente ici. Il nous dit que les gestes comportant le plus de grazia sont ceux qui se lèvent vers le haut. Ainsi justement notre sainte soulève du bout des doigts son manteau pour montrer son pied qui se prépare à quitter le sol. De l'autre main elle porte (à peine) son vase de parfum vers les fleurs qui représentent les odeurs qui s'en exhalent en apesanteur. La pointe du couvercle souligne ce mouvement vers le haut. Olivia Powell nous indique que les maitres de danse de la moitié du Quattrocento pensaient de la même façon, favorisant « un atto de aiereoso presenza et rilevato movimento colla propria persona $[. ..] »^{63}$ ( un geste aérien de présence et un mouvement élevé de sa propre personne »). À droite, quelques mèches s'envolent, mues encore une fois par une espèce de brise invisible, encore quasi-warburgienne. La survivance est pourtant moins celle d'une antique nymphe que de la Madeleine de Montefiore. Dans les deux cas la chevelure de la sainte est comme agitée par un souffle. L'on pense à celui qui éteint la chandelle dans la Pietà de Crivelli au Brera, et soulève encore une magnifique chevelure magdaléenne, l'Esprit Saint. Elle est en effet animée par la grâce divine.

Ici, point de ménade extatique de ses origines iconographiques ${ }^{64}$. Dans ses gestes on voit un écho aux mots de Léonard: «Les membres et le corps doivent s'adapter avec grâce à l'action que tu veux faire faire à la figure; si tu veux qu'elle soit jolie, fais-lui les membres élégants et déliés sans trop de muscles ${ }^{65}$ ». Si le sens du mouvement est subtil, c'est en raison de sa haute naissance. Une démarche naturelle mais harmonieuse et quelque peu stylisée caractérisait la danse des élites, qui régulait le corps, le maîtrisait. La danse curiale n'était pas ostentatoire ${ }^{66}$. Ici la cortegiana semble esquisser le sempio, simple pas vers l'avant ${ }^{67}$. En effet, dans les peintures contemporaines, une gestualité 
accusée dans la danse, de grands bonds par exemple, était l'apanage des femmes de basse extraction, ou encore celles qui font un mauvais usage de leur corps telle Salomé ${ }^{8}$. Guglielmo Ebreo se plaint des gens de basse extraction qui dansent lubriquement dans la convoitise charnelle. Il nous enjoint de danser avec virtù ${ }^{69}$ Étaient réservés à la dame de lignage, onesta, des gestes contenus, un maintien tout en retenue, avec ses vêtements qui ne montrent que peu les effets de son mouvement ${ }^{70}$. En effet, la discipline et le contrôle de la danse sont des techniques du corps qui domptent ainsi les instincts effrénés que la Madeleine a subis dans sa vie de pécheresse avant sa conversion à la vie de grâce ${ }^{71}$. Ainsi on perçoit, comme avec un œil quattrocentesque, les mouvements corporels de la Madeleine qui traduisent les mouvements de son âme, par une comparaison avec une gestualité de la danse mondaine et profane ${ }^{72}$. MarieMadeleine fait le lien entre le profane et le sacré, précisément à cause de sa vie avant la conversion. Par le biais de celle-ci, elle est souvent associée à la musique et la danse dans les contrées plus au nord. Le drame sacré de la Madeleine Digby, texte du xve siècle anglais, par exemple, la montre dansant à la limite du dévergondage. Plus tard, pour représenter un moment similaire de sa biographie, Lucas van Leyden gravera la danse de Marie-Madeleine. Lightbown fait justement l'hypothèse d'une inspiration septentrionale pour la Madeleine de Montefiore, et utilise une Madeleine de Lucas van Leyden comme exemple ${ }^{73}$. Il y eut en outre des danses nommées d'après la sainte pécheresse ${ }^{74}$. On peut d'ailleurs en trouver parfois des traces en Italie. Deux images trecentesques font accompagner la sainte par des instruments de musique conjugués avec le mouvement ${ }^{75}$. Celle de Spinello Aretino était un étendard utilisé en procession, donc en mouvement planifié ; celle de Luca di Tommè la montre en extase, soulevée par les anges, les boucles remués par l'animation céleste. Un lieu commun des traités de danse du Quattrocento est le rôle que tient la musique en réglant l'esprit ${ }^{76}$. Si cela peut paraître ténu, dans le récit anonyme du Quattrocento Conversione di Maria Maddalena et resurrezione di Lazzaro, la courtisane fustige sa sœur qui l'importune car elle a déjà préparé la fête en chanson, musique et danse $\mathrm{e}^{77}$. Toute cortigiana onestà d'ailleurs, surtout en milieu vénitien, était associée à la musique et donc à la danse ${ }^{78}$.

La voie qui rattache la Madeleine à la danse est aussi indirecte. Elle est souvent comparée à David son aïeul, par Ubertin de Casale par exemple, en ce qu'il fut adultère, et aussi en ce qu'il fut associé à l'onction ${ }^{79}$. Le cou de la belle est comme les tours du roi oint (la racine étymologique de son nom "Migdal» se rapporte en hébreu à son château, en référence à sa naissance noble ${ }^{80}$ ), selon le Cantique. David a aussi dansé devant l'arche de l'alliance. Le roi est invoqué comme exemple de danseur vertueux dans des traités de danse du Quattrocento ${ }^{81}$. Connue elle aussi pour sa danse sacrée, cette autre Marie, la sœur de Moïse, avatar de la Madeleine, est présente en pleine action, tambourin en main, dans la chapelle de la Madeleine à Assise. Chez les artistes italiens en général et Crivelli en particulier et plus spécifiquement en ce qui concerne la Madeleine de Carpegna, on peut parler de danse. Rappelons d'abord que Dürer, en visitant Venise, veut être gentilhomme-peintre comme les autres de son métier dans la Sérénissime. Il se met alors à apprendre à danser ${ }^{82}$. D'ailleurs l'importance de la danse dans les cours italiennes de cette époque ne peut pas être exagérée et c'est bien pour ce milieu que Crivelli peint en général. Cet artiste vénitien, chevalier honorifique, aurait-il lui aussi dansé, tout comme les artistes que Dürer voulait prendre en modèle ${ }^{83}$ ? En tout cas il est difficile d'imaginer que la comtesse de Carpegna, comme toute dame de cour, n'eût pas été entrainée à la danse depuis son plus jeune âge ${ }^{84}$. C'était une formation de longue durée qui apprenait à maintenir le corps même en dehors du temps de la 
musique - la mise en scène corporelle n'en finissait jamais ${ }^{85}$. Les minauderies magdaléennes exacerbent peut-être quelque peu cette façon d'apparaître, mais l'instruction qui en est la cause devait être bien familière à la comtesse et aux nobles familles de Carpegna qui venaient vénérer la sainte courtisane. Par ailleurs la danse, comme tous les accoutrements de la Madeleine traditionnelle, peuvent se lire de deux façons. Les habits sont ceux d'une courtisane ou simplement d'une dame de cour, ou bien d'une élue dans la cour céleste. Le parfum est celui qui attirait des amants ou bien celui qui fut offert au Seigneur, pour ensuite émaner de ses paroles prédicatrices. Les cheveux aussi sont un appât féminin ou bien un don de soi à Dieu et réciproquement un don de Dieu pour couvrir une nudité elle aussi ambiguë (fig. 4). Tout dispositif magdaléen fonctionne sur ce dédoublement du sens de ses signes iconographiques, de cette ambiguïté de signification. La danse, elle aussi, serait, soit impudique soit vertueuse, selon le caractère de celui ou celle qui s'y engage ${ }^{86}$.

Les balli étaient souvent organisés en lien avec la sorte de tournoi ${ }^{87}$ dont l'audience de Crivelli raffolait ${ }^{88}$, les dames y portaient leurs plus beaux atours, notamment des manches précieuses ${ }^{89}$. Les salles de danse étaient, tout comme la pièce où frétille notre Madeleine, tendus de beaux tissus ${ }^{90}$. Un lieu commun pour décrire les dames en bassa danza était de les regarder comme une vision venue droit du ciel ${ }^{91}$. Et cette Madeleine est bien une vision entr'aperçue de la cour céleste. Ses pieds se posent sur le sol marbré. Celui-ci est un avatar de ces panneaux analysés par G. Didi-Huberman qui figurent l'Incarnation christique, dont la Madeleine est une initiée intime ${ }^{92}$. Rougeâtre, il est aussi une rémanence de la Pierre de l'Onction, sur laquelle le Christ mort a reçu la myrrhe et l'aloès de la Madeleine ${ }^{93}$. Il n'est pas interdit d'y voir simplement, en même temps, le sol en marbre d'un palazzo, le support même des pas de danse ${ }^{94}$. Chez la sainte pécheresse en particulier, il n'y a aucune contradiction. Le sol et la plinthe marbrée d'ailleurs encadrent son pied et son mollet. Sa limite supérieure souligne précisément le genou fléchi pour le passo entamé, la répétition ondulante de séraphins en dessous semble représenter la suite du mouvement. Leur entrelacs qui se lèvent et puis retombent rappelle le pas chorégraphique de l'ondeggiare ${ }^{95}$. Quand le bout du pied quitte l'espace peint pour l'espace du spectateur, il pointe la signature sur un cartellino du peintre. Il fonctionne de la même manière que la main de Jean qui pointe le nom Giovanni sur le cartellino signé de la Pietà de Brera de Giovanni Bellini (fig. 15). C'était bien le jeu d'interaction entre le membre corporel du saint peint et le cartellino qui garantissait l'efficacité de l'image sacrée, voire miraculeuse " qui pourrait pleurer »96. Il est fort approprié qu'une peinture de la Pietà pleure. Ici, chez la sainte courtisane à la cour céleste, il s'agit d'une peinture qui pourrait danser. Or la danse est un art marqué par le contact, par le toucher. Jennifer Nevile a bien montré que le contact était un élément majeur de ces balli dont les participants et les spectateurs avaient une conscience aiguë ${ }^{97}$. Aussi, celles-ci mimaient les relations galantes et amoureuses entre homme et femme ${ }^{98}$, tout comme la Madeleine toute en leggiadria ici représentée avec son regard vezzoso. Ce dernier terme pouvait s'appliquer à une femme qui avait conscience de sa beautée9, autant que d'une peinture ${ }^{100}$. Parés de leur plus beaux atours, les danseurs tombaient dans les flammes de l'amour par le jeu des regards croisés ${ }^{101}$. Ce regard en coin de la sainte, ce regard qui attire et qui happe, che balestrava, est aussi un regard dont les paupières et les pupilles semblent descendre. En effet, les instructeurs de danse recommandaient aux jeunes demoiselles de bien garder leurs yeux baissés ${ }^{102}$. Mais si cette pala était installée à la hauteur d'un autel, l'œil de la sainte aurait tout à fait été en mesure de croiser celui ou celle qui viendrait la vénérer, la désirer, selon la 
vieille dynamique dévotionnelle franciscaine de la Madeleine. Les pastiglie, comme signes du va-et-vient votif, par le biais de la surface du tableau, aident à faire glisser la personne de la Madeleine dans l'espace du spectateur, de la dévote, de l'amoureux. Elle séduit celui ou celle qui vient la vénérer avec une invitation à toucher l'anse et le couvercle de son vase (on pourrait ajouter que si elle s'apprête à offrir son vase au Seigneur, la danse pouvait aussi être une mise en scène d'un cadeau - des épices odorantes par exemple - à offrir au seigneur $\left.{ }^{103}\right)$. Ces pastiglie grossissent au fur et à mesure de la carrière de Crivelli, et nous voilà en 1492 et vers la fin de sa vie en 1495. S'agit-il d'une invitation à saisir le corps de la sainte ? Comme l'anse du vase ? Défaire ces cheveux par ce diadème, défaire ces manches, cette boucle de ceinture, les boutons de son corsage? J'ai analysé ailleurs une longue tradition d'un corps à corps virtuel entre le spectateur courtois et la Madeleine en pied, mise en place dès $1285^{104}$. Cette Madeleine pourrait être une reformulation quattrocentesque de celle-ci. En tout état de cause, par le biais de sa manche, gage d'amour, par le biais de son diadème, par la fermeture de sa ceinture, les boutons de son corsage, elle semble inviter le spectateur à se joindre à elle dans cette danse de son ancêtre adultère David devant le tabernacle, dans une danse de cour d'une courtisane céleste.

\section{L'œil de Caterina}

34 Jusque-là nous avons eu une lecture présumant une approche, si je puis dire, hétéronormative de ces deux Madeleine qui séduisent un spectateur masculin par le jeu de leurs yeux et la mise en scène de leur corps. Or nous avons aussi beaucoup insisté sur la possibilité que ce soit une femme qui ait commandité la Madeleine carpegnate. L'œil du Quattrocento, selon Michael Baxandall, le fait a été souvent remarqué, est un œil normatif donc masculin par défaut. Quid donc d'un regard féminin surtout sur la Madeleine de 1492 ?

\section{Un regard féminin au Quattrocento}

Le sujet d'un regard spécifiquement féminin à la Renaissance mériterait sa propre étude, et un sujet aussi vaste ne peut être exploré à fond dans ces quelques pages. Il est malaisé, même aujourd'hui, de cerner quel est ce regard féminin en soi, qui serait plus ou moins libre du regard masculin normatif, lequel depuis la nuit des temps, a tant encadré les images de toutes sortes ${ }^{105}$. On pourrait bien sûr théoriser un regard féminin dans le cas peut-être d'une religieuse qui peint pour son propre couvent, telle Plautilla Nelli, par exemple. Mais en dehors d'un cas de figure de ce type, peut-on réellement parler d'un regard spécifiquement féminin sur l'image d'une femme? Traquer sa présence fugitive dans l'œil du Quattrocento risque l'anachronisme non-arassien. Il y a des terrains qui se sont révélés prometteurs : Les études du mécénat féminin ouvrent cette voie, mais ne se penchent pas forcément sur le comment du voir féminin. Certain'e's chercheur'se's ont bien relevé le défi. Victoria Ehrlich a porté son attention sur le regard féminin adressé à un contenu mythologique dans la peinture du Quattrocento ${ }^{106}$. Adrian W. B. Randolph a étudié les deschi da parto, par exemple, pour approfondir la notion en regardant des objets qui se destinaient à une audience de femmes et qui circulaient entre femmes. Il a raison d'appeler le sujet une terra incognita ${ }^{107}$, nous faisant part de ses doutes quant à la quête des styles cognitifs des hommes qui on produit ou les femmes qui ont manié et contemplé ces objets ${ }^{108}$. La 
question est d'autant plus épineuse ici que la femme représentée se doit d'être attirante pour les hommes, et qu'elle fut imaginée par un homme. Encore sa vita voulait-elle qu'elle ait péché avec ce même beau corps avec des hommes, donc fut un objet de leur convoitise par le biais de regard. Comment concilier tout cela avec la commanditaire de cette représentation? J'offrirai ici quelques possibilités de penser le regard féminin sur la Madeleine carpegnate: pensée et pratiques dévotes, pensée et pratiques vestimentaires, pratiques kinesthésiques.

Dans un premier temps, donc, parlons du bref passage chez Michael Baxandall qui aborde l'imagination dévote de la jeune fille. Des textes de nature religieuse imprimaient sur elle des pensées pieuses ${ }^{109}$. Or, un des buts des images des saint·e·s est d'inculquer de bons modèles aux âmes impressionnables qui les porteraient par la suite dans leur esprit et leur cœur pour inspirer la piété, les bonnes mœurs et actions. Il fallait entrer par sympathie dans l'image et en avoir le sentiment de bien connaître celles et ceux que la peuplent. Marie-Madeleine est nommée comme un de ces personnages. Cette attitude est ancrée de longue date, remontant aux pratiques dévotionnelles médiévales, particulièrement recommandées aux femmes en milieu franciscain en Italie ${ }^{110}$. Il n'est pas difficile de voir dans cette image de la Madeleine un modèle tout plein de grâces saintes et mondaines qui convenait à la contemplation de cette commanditaire, une comtesse, elle aussi donc dame de cour. La Madeleine semble incarner à elle seule toutes les qualités dont une femme doit se doter, que cela soit selon l'œil féminin selon le point de vue formulé par Alessandra Strozzi, qui cherche la virtù dans la grâce et la démarche ${ }^{111}$, ou qui seront un peu plus tard recommandées à la donna di palazzo par Baldassare Castiglione, dont la grâce est le leitmotiv ${ }^{112}$. Elle se tient donc comme un miroir idéal pour Caterina. Elle fonctionne comme une inspiration pour elle dans la vie de palais d'ici-bas qu'elle doit vivre en tant que comtesse, et dans l'au-delà pour un exemple de sainteté féminine. Il est vrai aussi que pour une femme mariée et mère, la Madeleine serait un modèle plus accessible pour Caterina qu'une quelconque vierge martyre. Comme la Madeleine, c'est une femme qui a connu le beau monde et tous ses plaisirs, le lit (en l'occurrence matrimonial de la commanditaire) inclus.

Suivant cette ligne de pensée nous pourrons aussi revenir sur la façon dont Crivelli souligne la réalité des accessoires de vêtements de cette Madeleine : boutons, boucle de ceinture, diadème, manche. Or ces parures ne sont pas toujours que des cadeaux d'un amant à sa dame telle la peccatrix dans sa vie antérieure, ni seulement des éléments de trousseau de mariage. Nous avons dit que ce sont, et Crivelli l'accentue par son usage des pastiglie a relievo, autant d'offrandes de dévôt-e's à une idole chrétienne, telles que Thomas Golsenne l'analyse. Pour ne prendre qu'un exemple citons la dame Ysabecta qui offrait des vêtements précieux à une statue de Jésus et l'autre Marie ${ }^{113}$. Une pratique apparentée serait celle des poupées saintes ${ }^{114}$. Tout comme les lectures dévotionnelles, les bambini étaient censés inculquer la piété chez la jeune fille à marier ${ }^{115}$. Bien que cet aspect soit primordial, il n'empêche que des vêtements, parfois de rechange même, faisaient partie de leur attrait ${ }^{116}$. On décrit les atours des petits personnages par exemple ainsi : " enfant vêtu de velours cramoisi, d'un petit manteau de brocard vert, avec les manches de sa chamarre brodées de perles ${ }^{117}$. On songe immanquablement aux parures crivellesques. En l'occurrence, au moins une version des ces poupées représentait Marie-Madeleine habillée de satin rouge, brodée de perles ${ }^{118}$. Aux côtés des belles manches, les poupées se trouvaient dans les trousseaux 
des jeunes filles et se passaient de mère en fille ${ }^{119}$. Les agréments de la toilette de la Madeleine en relief rappellent l'acte cultuel d'habiller les figures des saintes images autant que les saintes poupées ${ }^{120}$. Ces gestes dévotionnels revenaient, d'ailleurs, souvent aux mains des femmes ${ }^{121}$. Quand il s'agit d'une poupée-statuette de la Vierge, l'autre Marie, on lui boucle les cheveux ${ }^{122}$, on la parfume même ${ }^{123}$. Ce n'est pas sans rappeler la toilette - au sens inclusif de soins de visage, de parfum, de parure - de la bien soignée Madeleine carpegnate. Et ce même genre de toilette - des atours somptueux à la chevelure coiffée, le tout parfumé - aurait bien correspondu au genre de celles de la Comtesse de Carpegna. Richard Trexler n'hésite d'ailleurs pas à dire de ces jeune filles du grand monde, parées pour paraître en vêtements coûtant qui sait combien de florins, qu'elles étaient elles-mêmes autant de poupées sacrées ${ }^{124}$.

Le lien entre femmes et vêtements est fort étroit dans l'anthropologie de cette société depuis bien de siècles déjà. Dans la pensée chrétienne, n'est-ce pas de la faute d'Ėve si on les porte, se découvrant nu'e's ? Il revenait à la mère de l'espèce humaine de filer la laine en tant que punition pour sa faute, au même titre que d'enfanter dans la douleur. Dans l'imagerie mariale, souvent la Mère de Dieu, elle aussi, file. Cette association allait de soi puisque la mère tisse l'enfant dans son ventre et le Christ a pris sa chair à elle comme un vêtement ${ }^{125}$. Une telle notion s'insérait bien dans la pensée paulinienne qui parle du corps de péché et du corps glorieux en en nous exhortant à se dévêtir de l'un et à revêtir l'autre ${ }^{126}$. L'équivalence corps-étoffe sera particulièrement reprise et développée par les Franciscains ${ }^{127}$. On compte bien des survivances de cette association jusqu'à aujourd'hui dans le mot "tissu » qui désigne à la fois le drap et le réseau de cellules qui composent un organe. Or dans la pensée de cette société la femme était au corps ce que l'homme était à l'esprit. Un regard féminin aurait des rapports avec l'étoffe que ne comprendrait pas un homme. Ce lien entre la femme et le vêtement allait plus loin dans le vécu quotidien. Pour Castiglione la donna di palazzo se devait de savoir s'habiller pour se mettre en valeur, mais le courtisan constate aussi que les femmes sont naturellement douées pour les arrangements de leur toilette ${ }^{128}$. Les hommes se plaignaient des dépenses vestimentaires pour leur jeunes femmes et les lois somptuaires s'en mêlaient. Or Marie-Madeleine particulièrement, en tant que femme vezzosa, coquette, était l'exemple-type de la femme trop prise (selon les estimations masculines) par le plaisir de ses vêtements, le péché de la vanité129, inhérent à la faiblesse innée du genre féminin en général. Et pourtant il fallait qu'une femme du monde les portent comme signes de sa propre valeur sociale ${ }^{130}$. Une femme de l'élite devait savoir très bien estimer la valeur liquide des atours propre à son sexe. L'on songe à Alessandra Strozzi qui estime le prix des vêtements de mariage de sa fille. Après une description détaillée elle finit avec ce constat: "Quand elle sortira elle aura plus de 1400 florins sur le dos $»^{131}$. La mère avait raison de son acuité. En effet, il en allait de la survie économique de la mariée ${ }^{132}$. Qui plus est, si le drap se faisait couper et tailler par un homme, le plus souvent les broderies et fanfreluches (rose, ceintures, boutons) qui caractérisent les atours de cette Madeleine étaient le produit des mains féminines ${ }^{133}$. Un œil de femme donc était bien à même d'apprécier, au sens large, la parure élégante dont Crivelli dote sa peccatrix sainte et courtoise.

Si la commanditaire n'avait vraisemblablement pas eu la culture mathématique pour mesurer les subtilités de la géométrie albertienne ${ }^{134}$, elle aurait par contre bien su apprécier une dimension kinesthétique dans cette Madeleine que je vois dansante. Ses atours sont ceux de la grande fête de cour et dans ce sens-là aussi, Caterina aurait pu se voir reflétée dans cette "Maddalena», dans sa gestuelle de danse de palais, 
gracieusement retenue, des gestes allant vers le haut, comme le recommandait Gugliemo Ebreo. Olivia Powell cite David Freedberg qui parle d'un «engagement spectatoriel senti ", une réponse physique, corporelle, à une image et à la gestuelle de ses figures. ${ }^{135}$ La comtesse aurait senti ces gestes de par son long entrainement, depuis sa petite enfance, à l'art chorégraphique. Aussi, bien que la plupart des balli curiaux fussent surtout des imitations des rituels de l'amour entre homme et femme, encore ne faut-il pas projeter une réalité littérale du bal sur le panneau. Les peintres étaient euxmêmes bien à même d'imaginer et d'étendre le champ pictural de la chorégraphie. Sharon Fermor par exemple insistent longuement sur ce point ${ }^{136}$. De plus, ne sont-ce pas les trois Grâces de Botticelli, trois femmes qui dansent ensemble, qui sont l'exemple-type de la danse interprété par les arts visuels du Quattrocento? La Madeleine carpegnate pourrait aussi très bien se préparer à un ballo in due avec Caterina. Du reste, poupées, vêtements, et danse - tout comme les deschi da parto - ont pour trait commun l'aspect tactile, ou comme le diraient Adrian W. B. Randolph et Olivia Powell, la kinesthésie de cette perception, ancrée dans l'expérience corporelle. On prend son'sa partenaire par la main ; on materne ces bambini ; on passe de main and main les deschi ; on ouvre les cassoni ; on manie les ailes d'une triptyque domestique ${ }^{137}$. Un vêtement est porté par le corps, couvre le corps, est, en quelque sorte, la chair ; un tissu est forcément textural, perçu par les nerfs de la peau, autre tissu. Cela concorde aussi avec la spiritualité médiévale féminine vécu somatiquement, étant donnés les présupposés postulant que la femme est à la chair ce que l'homme est à l'esprit ${ }^{138}$. Il allait de soi dans la pensée de l'époque que l'expérience féminine du divin est ancrée dans le vécu du corps et ses sensations physiques. Pour ce qu'il en est de la peinture étudiée ici, en Italie ces phénomènes se situaient surtout dans un milieu franciscain et les femmes mystiques qui les éprouvaient étaient souvent appelées des «secondes Madeleine ». Or nous avons plus haut cité Thomas Golsenne qui insiste justement sur l'aspect haptique de l'art de Crivelli, qui fait transférer le voir dans le toucher par le biais de ses pastliglie a relievo. Il est possible que de pareilles pratiques artistiques puissent donc faire particulièrement appel à un style de cognition visuelle qui corresponde à une expérience féminine.

\section{Et la séduction de ce regard aguicheur?}

Même sur les deschi da parto on trouve l'œil évaluatif d'un Pâris devant les trois déesses qui les juge selon leur beauté telle qu'elle s'accorde à son œil à lui ${ }^{139}$. Nous sommes pleinement confrontée's au devoir de la femme du monde d'être, avant tout, belle pour le regard de l'homme, telle que le préconise Castiglione. Une trace de l'œil féminin peut se retrouver par exemple dans les lettres d'Alessandra Strozzi au milieu du siècle. Mais la matrone dévisage les jeunes femmes pour les jauger par les critères de grâce et de beauté mis en vigueur par des hommes, dont Castiglione se fera l'écho ${ }^{140}$. Par ailleurs, les jeunes femmes ainsi étudiées le sont en tant qu'épouses potentielles pour le fils Strozzi. Elles se montrent dans le devoir d'être désirables, de se voir ainsi valorisées à travers un objectif dominant et prescripteur, donc réfractées à travers le regard de l'homme. Le regard masculin s'est greffé en quelque sorte sur le regard féminin à travers les siècles comme regard normatif. On sait que quelques décennies plus tard dans un contexte bien différent, il est vrai - un autre peintre vénitien s'adressant à un regard féminin n'hésite pas a accentuer tout ce qu'un regard d'homme aurait trouvé de plus agréable à contempler dans son lit. Je pense bien sûr aux analyses bien connues de 
Rona Goffen sur la Venus d'Urbino de Titien ${ }^{141}$. La chercheuse américaine a établi que dans la chambre nuptiale cette figure était là pour - entre autres - inspirer la jeune épouse à se préparer manuellement aux relations conjugales pour mieux se rendre fertile. Il y a un monde qui sépare les deux images, évidemment (une chambre d'époux de 1538 n'est pas une église de 1492) et pourtant il est pertinent de soulever que ce qu'un peintre pense va interpeller une femme. Titien lui présente une image-type de la femme sexualisée exprimée dans les codes hétéronormatifs. Adrian W. B. Randolph a repéré dans les deschi da parto, destinés spécifiquement à des spectatrices, des images de Vénus chargées de son pouvoir érotique et les regards de son public qui sont définis par le voir au masculin ${ }^{142}$. Par ailleurs, dans un paragraphe précédent nous avons suggéré que la Madeleine carpegnate agit comme miroir pour la comtesse. Un genre de toilette de cour comparable entre les deux, un maintien discipliné et gracieux, entrainé par la formation pour la danse, permettait peut-être à la comtesse de se voir réfléchie dans l'image de la courtisane céleste. Or, la donna di palazzo, bien plus que le cortegiano, se devait d'être attirante, désirable, ce aux yeux des hommes, par des critères que ceuxci définissaient. Sa virtù se déclinait sur les codes du genre et se basait sur l'investissement dans ses attraits physiques, bien que souvent spiritualisés dans la notion de "grazia» si importante, on l'a vu, pour cette Madeleine. Se voir en elle en tant que désirable, avec les charmes d'une femme du monde accentués, autant que les qualités plus éphémères et intangibles telles que la grazia, leggiadria ${ }^{143}$, serait se retrouver elle-même valorisée selon les normes mises en place par la population dominante de la société.

\section{L'œil de Carlo Crivelli ?}

41 N'oublions pourtant pas que l'artiste, lui, est un homme, et un homme par le peu qu'on sait de lui, sensible aux attraits des femmes, assez pour enfreindre la loi et enlever la femme d'un autre, bravant l'exil pour elle. Qu'il ait conféré tant de charmes à cette Madeleine toute vezzosa, en ferait-il un Pygmalion devant Galatée? Une dizaine d'années après que Crivelli eut peint cette Madeleine, Léonard de Vinci écrit «[...] le peintre contraint les esprits des hommes à tomber amoureux et à aimer une peinture qui ne représente aucune femme vivante. Et il m'est arrivé de faire une image à sujet religieux, achetée par un amant qui voulait en faire enlever les attributs de la divinité, pour pouvoir l'embrasser sans reproche. $»^{144}$ Que Crivelli ait été sensible à "l'appel amoureux " que décrit Daniel Arasse, à propos de certaines images saintes de Leonard ${ }^{145}$, de sa propre peinture, doit rester de la pure spéculation, mais il a bien produit une Madeleine qui serait une idéal de la beauté féminine plaisante pour n'importe quel regard masculin. Il y a en tout cas certainement quelque chose qui dégage d'elle un «appel amoureux». On serait pardonné si l'on ressent que Carlo Crivelli s'est pris au jeu.

\section{Conclusion}

Du strip-tease à Montefiore à une invitation à un ballo in due à Carpegna, les courtisanes célestes de Carlo Crivelli sont des inventions ingénieuses. Ses Madeleine interpellent lela spectateurrice dévotee, voire amoureux le cas échéant, soit par ses facultés optiques soit par ses facultés haptiques. Dans les deux cas sa faculté olfactive 
imaginaire autant que tactile sont absolument manifestées et ceci selon un dispositif mis en place depuis le Duecento dans le milieu franciscain, celui de son iconographie foisonnante. C'est l'époque aussi du dispositif de l'érotique courtoise quant à la formulation plastique de ce personnage dans cette même mouvance. Deux siècles plus tard, on voit un ressort similaire: Madeleine utilise ses charmes féminins, transsubstantiés mais ô combien reconnaissables, pour attirer son dévot-amoureux vers le Christ. Pourtant la part d'une commanditaire n'est pas négligée : les tendances artistiques de Crivelli vers le tactile et la somptuosité vestimentaire avaient tout pour plaire à une spectatrice. D'autant plus une dame mondaine telle que Caterina di Carpegna qui pouvait virtuellement entrer en participation avec celle qui mène le bal avec tant de grâce. Aussi n'est-il pas étonnant que le peintre vénitien exilé lui-même pour une affaire de péché de chair soit un interprète si éloquent de Marie-Madeleine et de sa façon de retourner de telles transgressions en toute beauté dans le droit chemin de Dieu.

\section{NOTES}

1. Pietro ZAMPETTI, Carlo Crivelli, Florence, Nardini, 1986, p. 15, 311-12.

2. Mostra della pittura veneta nelle Marche. Catalogo a cura di Pietro ZAMPETTI. Aug.-Sept. 1950. [Ancona. Palazzo degli Anziani.]

3. Thomas GOLSENNE, Carlo Crivelli et le matérialisme mystique du Quattrocento, Rennes, Presses Universitaires de Rennes, 2017. Sur le prestige de ses mécènes, voir pages 27-38.

4. Ibid., p. 27.

5. Janet ROBSON, «Pilgrim's Progress: Re-interpreting the Trecento Fresco Programme in the Lower Church at Assisi ", in The Art of the Franciscan Order in Italy (The Medieval Franciscans, vol. 1), ed. William R. Cook, 2005 p. 39-70.

6. Ronald LightBown, Carlo Crivelli, New Haven \& London, Yale University Press, 2004, p. 50-51.

7. Bien qu'on soit loin du monde des humanistes florentins, l'on songe à la "brise invisible " d'Aby Warburg, et le prolongement de sa pensée par Georges DIDI-HUBERMAN, "The imaginary breeze: remarks on the air of the Quattrocento ", Journal of Visual Culture, vol. 2, issue 3, décembre 2003, p. 275-289.

8. Ronald LIGHTBOWN, p. 195.

9. Sur cette mise en abyme : Thomas GOLSENNE, p. 216.

10. GRÉGOIRE LE GRAND, Homélies sur l'Évangile, t. 2, Homélies XXI-XL, éd. et trad. Raymond Etaix, Georges Blanc, Bruno Judic, Paris, Le Cerf (Sources chrétiennes, 522), 2008, Homélie XXV, p. 103-139.

11. Une référence au phénix se trouve sinon liée directement à la Madeleine par Bernard de Clairvaux, au moins dans son hymne aux Filles de Sion qui lui sont liées, cité par Alain MICHEL, Théologiens et mystiques au Moyen Âge, Paris, Gallimard, 1997, p. 280. Au début du Cinquecento, Antonio alAmanni évoque également l'oiseau dans sa Commedia de la Conversione de Maria Maddalena, ed. Pierre Jodogne, Bologne, Commissione per i testi di lingua, 1977, p. 19.

12. HÉRODOTE, Histoires, II, 72, voir l'édition de Philippe-Ernest Legrand, Paris, Belles Lettres, 1936, 1944, p. 114-115.

Images Re-vues, 16 | 2019 
13. "Primum fuit unguentum Magdalene de qua dicitur Io. XII quod domus impleta est ex odore unguentum sancte peccatricis; quia odor penitentie spirat usque ad supercelestes mansiones spirituum bonorum ut sit gaudium angelis dei super uno peccatore penitentia agente etc. Vide quod angelis est gaudium ad exaltationem, sed hominibus est exemplum ad correptionem; et fit de herbis amoris que in nostro habetur hortulo quas facile invenit homo iniquitates suas sine dissimilatione recogitat, cum istis herbis amaris ad ignem ferventis amoris aromata misceantur. Myrrha inquit sponsa Cant(ico) iiii et aloe cum omnibus primis unguentis (= Cant. 4, 14) utrumque autem amarissimum et a perservandis corruptionibus aptum et duplicem amaritudinem in penitente. Prima est amaritudo mortis cum incertitudine hore. Secunda est incertitudo (corr. certitudo) iuditii cum incertitudine sententie, ex quibus amarum conficitur et pungitivum unguentu ", UBERTINO DA CASALE, Arbor vitae crucifixae Jesu, Venise, A. De Bonetis, 1485 (incunable), édition facsimilé C.T. Davies, Turin Bottega d'Erasmo, 1961, p. 273.

14. $1: 3$ : «Mon bien-aimé est un sachet de myrrhe, qui repose entre mes seins.» (« fasciculus murrae dilectus meus mihi inter ubera mea commorabitur »).

15. Daniel Russo, « Entre Christ et la Madeleine », in E. Duperray, Marie Madeleine dans la mystique, les arts et les lettres. Actes du Colloque international (Avignon, 20-22 juillet 1988), Ève Duperray (éd.), Préface de G. Duby, Postface de Ch. Pietri. Paris, Beauchesne, 1989, p. 173-190.

16. Dans Courtly Love Undressed: Reading Through Clothes in Medieval French Culture, Philadelphia, University of Pennsylvania Press, 2002 p. 6 , E. Jane BURNS relève que depuis le temps des romans chevaleresques une manche peut figurer l'armoirie même de celle qui la porte, et , en plus un soi désirant.

17. Elle est déjà figure de la Sponsa chez Hippolyte de Rome et Origène.

18. Carole COLLIER FRICK, Dressing Renaissance Florence: Families, Fortunes, and Fine Clothing, Baltimore, Johns Hopkins Press, 2002, ; Christiane KLAPISCH-ZUBER, « Le complexe de Griselda. Dot et dons de mariage au Quattrocento » Mélanges de l'Ecole Française de Rome, 1982, 94-1, p. 7-43.

19. E. Jane BURNS, Courtly Love Undressed, p. 4-11.

20. Thomas GOLSENNE, p. 25-27.

21. Une recette à parfumer les vêtements et la camisa ou camiccia («chemise »), comme porte cette Madeleine en sous-vêtement, se trouve dans Giovantura ROSETTI qui donne plusieurs recettes pour arriver à différentes tonalités de blondeur, dans son Notandissimi secreti de l'arte profumatoria, éd. Franco Brunello et Franca Facchetti, Vicenza, Neri Pozza, 1973, p. 81.

22. Matthieu $26: 7$; Marc $14: 3$; Luc $7: 37$.

23. Thomas GOLSENNE, p. 83-89.

24. Dominique IOGNA-PRAT, "La Madeleine du Sermo in ueneratione sanctae Mariae Magdalenae attribué à Odon de Cluny » dans Mélanges de l'École Française de Rome - Moyen Âge, 85, 1992, p. 37-70. 25. Je me permets de renvoyer à ma thèse, Le Corps de Marie-Madeleine, à paraître ; pour la maison remplie de l'odeur de nard, voir Jean $12: 3$.

26. JACQUES DE VORAGINE, La Légende dorée, A. Boureau et al. trad., Paris, Gallimard (Pléiade), 2004, p. 512.

27. Giunta BeVegnati, O.F.M., Légende de la Vie et des Miracles de Sainte Marguerite de Cortone, tr. Mgr Luquet, Paris, Poussielgue-Rusand, 1859, p. 429.

28. Jean-Pierre ALBERT, Odeurs de Sainteté: la mythologie chrétienne des aromates, Paris, Ed. de l'EHESS, 1996, p. 174-177.

29. Je me permets de renvoyer à ma thèse, Le Corps de Marie Madeleine, à paraître.

30. Et il s'agit bien d'une relique qui existe à la ville sœur de Venise, Constantinople, et sera ramenée à la Sérénissime quelques années plus tard en 1476.

31. Hymnae Ecclesiae, ed. John Henry Newman, London, MacMillan, 1865, p. 358 : « Ex lebete facta phiala / De luto luci reddita / in vas translata gloriae ». Voir aussi Katherine L. JANSEN, The Making of the Magdalen: Preaching and Popular Devotion in the Later Middle Ages, Princeton, Princeton University Press, 1999. p. 159., p. 173 n. 24 : «tales lebes fuit Magdalena que ardore succensa libidinis 
multum exhibuit et in eam tot dyabolus coxit cibos quot peccatores in eius amore succendit [...] hoc lebes multos suo contactu polluit ", ms Assisi 470 f. 494v; ibid., p. 243 n. 147 : « qui vas perditum vas ire vas contumeliae transtulit et transformuit in vas gloriae ", ms Venice, Marc. lat. fondo antico, 91, 1775, $\mathrm{f}$. $16 \mathrm{v}$, cité aussi par Élisabeth PINTO-MATHIEU, Marie-Madeleine dans la Littérature du Moyen Âge, Paris, Beauchesne, 1997, p. 46. Pour l'équivalence entre Marie-Madeleine et son vase je me permets de renvoyer au chapitre 10 de ma thèse, Le Corps de Marie Madeleine, à paraître.

32. Thomas GOLSENNE, p. 114-119.

33. On peut suivre les fortunes de la Madeleine angevine chez V. SAXER, Le Culte de MarieMadeleine en Occident: Des Origines À la Fin Du Moyen Âge, Publications de la Société des Fouilles Archéologiques et des Monuments Historiques de l'Yonne, 1959. Une autre inflexion à la Madeleine angevine se trouve dans ma thèse, Le Corps de Marie-Madeleine, à paraître.

34. Thomas GOLSENNE, p. 57-58.

35. Ibid., d'après Tomei, p. 27.

36. Paul HILLS, "Venetian Glass and Renaissance Self-Fashioning", in Concepts of Beauty in Renaissance Art, ed. Fr. Ames-Lewis et M. Rogers, Ashgate, Aldershot, 1998, p. 163-178.

37. Entre 1465 et 1475 , on pouvait en fait s'approvisionner en pigments comme en parfums, et même en verre, chez le même marchand. A. MozzATo, "Oppio, triaca e altre spezie officinali a Venezia nella seconda metà del Quattrocento ", in Venice and the Veneto during the Renaissance: the Legacy of Benjamin Kohl, a cura di M. Knapton, Jennifer E. Law, A. A. Smith, Firenze, Reti Medievali/Firenze University Press, 2014, p. 155-184.

38. «Verum Maria, ubi nubiles subiit annos, formositate corporis, pulcherrima splendens, speciosa nimis, enituit, decens membrorum ductu, vultu venusta, mira caesarie, lepore gratiosissima, melliflua mente ; cujus oris decor et gratia labiorum ( $\operatorname{Pr} 22,2)$ ut mistus rosis candor liliorum. Formae denique et pulchritudinis gratia tant resplenduit, ut singulare, atque mirificum opificis Dei diceretur figmentum » (Patrologie Latine, éd. J-P Migne Paris, 1852, t. CXII col. 1433-34.

39. JACQUES DE VORAGINE, p. 510.

40. Ronald LIGHTBOwN (p.194) s'attarde sur cet élément de son iconographie, et Marilena mosco la qualifie de «civetteria » : "L'Iconografia della Maddalena nella pittura di Carlo Crivelli dalla Pala di Montefiore a quella di Fabriano ", in Il Patrimonio disperso : il "caso" esemplare di Carlo Crivelli, Actes du colloque, Montefiore dell'Aso, Camerino, Porto San Giorgio, 12, 26 oct., et 9 nov., a cura di Marina Massa, Regione Marche, Assessorato alla Cultura Centro Beni Culturale, San Severino Marche, Maroni, 1999, p. 83-112.

41. Thomas GOLSENNE, p. 110-113.

42. Cité dans Amélie BERNAZZANI, Un seul corps : La Vierge, Madeleine et Jean dans les Lamentations italiennes (ca. 1272-1578), Tours, Presses universitaires François-Rabelais, 2014, p. 126.

43. PSEUDO-DENYS L'ARÉOPAGITE, Lettres, IX, 1, 1104, cité par Georges DIDI-HUBERMAN, Fra Angelico : dissemblance et figuration, Paris, Flammarian (Champs), 1990, 1995, p. 87.

44. Francesco Vittorio LOMBARDI, "Una 'maddalena' di Carlo Crivelli per i conti de Carpegna », Studi Montefeltrani, $\mathrm{n}^{\circ}$ 9, p. 21-35.

45. La Madeleine, selon une Vita de mouvance cistercienne du XII ${ }^{\mathrm{e}}$ siècle était elle aussi comtesse Élisabeth PINTO-MATHIEU, Marie Madeleine, p. 139-148.

46. Ce maquillage - trait noir sur un regard « pervers » qu'aurait dénigré Yves Bonnefoy - peut se lire comme une méta-peinture crivellesque. Thomas Golsenne de dire: «La peinture est un maquillage et réciproquement. Je propose de voir dans le maquillage une métaphore de l'art humain en général », p. 45. Cette espèce d'« eyeliner » quattrocentesque souligne cet œil étiré, forme qui signale, selon Bartolommeo Fazio, l'immoralité. Cité par Michael BAXANDALL, L'Cil du Quattrocento. L'Usage de la peinture dans l'Italie de la Renaissance, trad. Y. Delsaut, Paris, Gallimard, 1985, p. 93.

47. Voir plus haut, note 7 . 
48. Dans les pratiques réelles les dames parfumait leur chevelure. Evelyn WELCH, «Scented Buttons and Perfumed Gloves: Smelling Things in Renaissance Italy ", in Ornamentalism : The Art of Renaissance Accessories, ed. Bella Mirabella, Anne Arbor, University of Michigan Press, 2011, p. 13-39, p. 22.

49. S'agit-il d'une fleur pourvue d'un symbolisme spécifique, comme le chardon pour la Passion (je remercie Susan Hirshberg pour cette suggestion)? Cela s'harmoniserait avec le collier en corail qui renverrait aussi aux plaies christiques. Serait-ce une fleur céleste composite ? Une fleur biblique telle qu'imaginée par un Italien qui ne s'est jamais rendu en Terre Sainte? En tout cas, Thomas GOLSENNE va plus loin dans cette pensée sur le végétalisation de la présence divine dans une perspective de " panthéisme franciscain », voir p. 189-197.

50. Evelyn WELCH, «Scented Buttons », p. 13-39.

51. Thomas Golsenne a très bien démontré comment ces pastiglie a relievo ne sont pas des régressions vers une plasticité toute "médiévale ». Il fait le lien avec les statues de culte auxquelles on offre autant d'habits et d'accessoires de parure. Thomas GOLSENNE, p. 146-151.

52. Ibid., p. 192-193.

53. Voir chapitre $\mathrm{X}$ de ma thèse, à paraître Le Corps de Marie-Madeleine.

54. Je me permets de renvoyer à Vicki-Marie PETRICK, «Unctio : la peinture comme sacrement dans la Pietà de Giovanni Bellini à la Pinacothèque Vaticane ", Images Re-vues, 9, 2012 [en ligne] https://journals.openedition.org/imagesrevues/1899. Consulté le 28/07/19.

55. Daniel Russo, « Saint François, les franciscains et les représentations du Christ sur la croix en Ombrie au XIII ${ }^{\mathrm{e}}$ siècle : recherches sur la formation d'une image et sur une sensibilité esthétique au Moyen Age ", Mélanges de l'École française de Rome - Moyen Âge, 96, 1984, p. 647-717.

56. Thomas GOLSENNE, p. 204.

57. Dans ces mêmes années 1489-90, Léonard présente une torsion du corps de la cortigiana maîtresse qui se laisse surprendre en un début de mouvement amoureux. Daniel ARASSE, Léonard de Vinci : le rythme du monde, Paris, Hazan, 1997, p. 397.

58. Voir note 38.

59. Michael BAXANDALL, p. 120-126.

60. Olivia POWELL, The Choreographic Imagination in Renaissance Art, thèse doctorale, Columbia University, 2012 ; Sharon FERMOR, « On the Question of Pictorial 'Evidence' for Fifteenth-Century Dance Technique ", Dance Research: The Journal of the Society for Dance Research, Vol. 5, No. 2(Autumn, 1987), p. 18-32; Jennifer NEvILE, The Eloquent Body: Dance and Humanist Culture in Fifteenth Century Italy, Indianapolis, Indiana University Press, 2004

61. Pour Thomas GOLSENNE, les théories d'Alberti vont « dans un sens divergent de l'esthétique crivellesque ; parfois dans le même sens », p. 55.

62. Olivia POWELL, p. 8-16.

63. Ibid., p. 25, Powell cite Guglielmo EBREO, De practica sue arte tripudii, trans. Barbara Sparti (Oxford: Clarendon Press, 1993), p. 96-97.

64. On peut se référer par exemple à la Madeleine de la Crucifixion de Cimabue dans l'Église supérieure d'Assise. Voir chapitre III de ma thèse à paraître, Le Corps de la Madeleine,

65. Michael BAXANDALL, p. 196, cité ici d'après la traduction d'André Chastel dans Léonard de Vinci, Traité de la Peinture, textes réunis, traduits et annotés par André Chastel, Paris, Hermann, 1964, 2004, p. 140.

66. Olivia POWELL, p. 134-135.

67. SharOn FERMOR, p. 25.

68. Je remercie Thomas Golsenne pour cette observation. Par ailleurs, un cycle trecentesque de la Vie de Marie-Madeleine aux murs de sa chapelle au Bargello contraste notre sainte avec Salomé, presque comme des exemples du bon et mauvais usage du corps féminin, voir chapitre VI de ma thèse à paraître, Le Corps de la Madeleine. 
69. Cité dans Olivia POWELL, p. 144 et n. 51

70. Jennifer NEVILE, p. 98.

71. Et je suggérerais que ces boucles parfaites et l'épilation du front et des sourcils sont autant de signes d'un corps bien discipliné.

72. Michael BAXANDALL, p. 95.

73. Ronald LightBown, p. 573.

74. H. Colin SLIM, «Mary Magdalene, Musician and Dancer, " in Early Music, October, 1980, p. 460-473.

75. Ibid., p. 461-462.

76. SharON FERMOR, p. 2.

77. Katherine L. JANSEN, The Making, p. 159.

78. The Courtesan's Arts: Cross-Cultural Perspectives, ed. Martha FELDMAN et Bonnie GORDON, Oxford, Oxford University Press, 2006.

79. " Hoc est unguentum quo David tribus vicibus mystice est inunctus: primo in Bethlehem in domo patris sui, I Regum 16, 13; secundo in Hebron super Iudam, II Regum 2, 4; tertio super omnes tribus, iterum in Hebron, II Reg. cap. 5, 3. Quid David, qui 'manus fortis' interpretatur, convenientius significare potest quam fortem spiritum Magdalenae, qui quidem unguento ferventis poenitentiae tripliciter inunctus fuit? : "Secundum fuit ungentum strenuitatis et iustitie quo unctus est David: manufortis et pulchro aspectu, de quo dicitur in psalmo: Inveni David servum meum etc [Ps. 89, 21]. Hoc ungentum conficitur ex oleo et balsamo. Oleum lucet et balsamum redolet, quia ex operibus iustitie procedit lucida conscientia et odorifera fama, ut provideamus bona, non solum coram Deo sed etiam coram omnibus hominibus ", UBERTINO DA CASALE, Arbor, p. 273.

80. Cette étymologie remonte à Jérôme : Dominique IOGNA-PRAT, p. 44-49.

81. Jennifer NEVILE, The Eloquent Body, p. 65.

82. Ibid., p. 24.

83. La première trace écrite de son titre de " equus » date de 1490, justement: Thomas GolSENNE, p. 35.

84. Jennifer NEVILE montre des jeune filles de l'élite entamant un apprentissage en danse de cour dès sept et dix ans, en 1460, p. 24.

85. Ibid. p. $45,57$.

86. Ibid., p. 92-93.

87. Ibid., p. 43.

88. Thomas GOLSENNE, p. 105.

89. Jennifer NEVILE, p. 40.

90. Ibid., p. 37.

91. Ibid., p. 19, 22 et 53.

92. G. DIDI-HUBERMAN Fra Angelico: Dissemblance et figuration, Paris, Flammarian (Champs), 1990, 1995 . La dilectio professionis de la sainte est décrite comme telle dans le Sermo du pseudo-Odon de Cluny, voir Dominique IOGNA-PRAT, p. 46.

93. Andreas PRATER, "Mantegnas Cristo in Scurto", Zeitschrift für Kunstgeschichte, 48, 1985, p. 285-292 ; Gioia MORI, « Quarto fuit sanguinisa deitate : la disputa di S. Giacomo della Marca nel Polittico di Massa Fermana di Carlo Crivelli », Storia dell'arte, 47, 1983, p. 17-27; Georges DIDIhuBERMAn, Fra Angelico, p.143. Thomas GolSENNE aborde le marbre rouge de Crivelli dans ce contexte, p. 91-94.

94. Cet argument serait à nuancer avec les pages de Paul HILLs sur les sols des palazzi vénitiens, qui toutefois se faisaient eux aussi, oindre! Venetian Colour: Marble, Mosaic, Painting and Glass 1250-1550, Yale University Press, 1999 p. 81.

95. Sharon FERMOR, p. $25:$ : [...] in which the dancer rises on to the toes on making a step and sinks gracefully down at the end. » 
96. Daniel ARASSE, "Giovanni Bellini et les limites de la mimesis : la Pietà de Brera ", dans Künstlerische Austausch/Artistic Exchange, Akten des XXVIII Internationalen Kongresses für Kunstgeschichte, Berlin 15-20 Juli 1992, Berlin, Akademie Verlag, 1993, p. 503-509.

97. Jennifer NEVILE, p. 15-16.

98. Ibid., p. 28-31 et 53-55. Une danse, particulièrement appropriée pour notre sujet, était la Merçantia, qui mettait en scène un femme qui allait d'amant en amant.

99. Ibid., p. 53.

100. Michael BAXANDALL, p. 195-96. Les instructeurs de danse et les humanistes discutaient de l'art visuel et de l'art chorégraphique en termes parallèles : Jennifer NEVILE, p. 69-74.

101. Ibid., p. 53-54.

102. Ibid., p. 88 .

103. Ibid., p. 37-38.

104. Voir chapitre IV de ma thèse, Le Corps de Marie-Madeleine, à paraitre.

105. La tentative de cerner un regard exclusivement féminin n'est qu'émergent avec une nouvelle génération de cinéastes femmes. En 2018 seulement, le Film Society at Lincoln Center a présenté une collection de films qui tente d'explorer le concept, nommé précisément The Female Gaze. Ce regard est en devenir, se conjugue au présent et au futur.

106. Des études se préparent de cette chercheuse depuis sa thèse récente et sa maîtrise en amont qui se penchait sur le « voir genré » voir Victoria EHRLICH, Perception and Presentation: Mythological Imagery and the Female Gaze in Italian Renaissance Art, mémoire de Master, University of Texas, Arlington, 2007.

107. Adrian W. B. RANDOLPH, «Gendering the Period Eye : Deschi da parto and Renaissance Visual Cuture ", Art History, vol. 27, n4, Septembre, 2004, p. 538-562: " My aim is not only to explore the largely terra incognita of women's visual culture, but also to inquire as to how visuality was structured by gender, among other things », p. 542.

108. Adrian W. B. RANDOLPH : "I am not at all certain that one can recuperate the 'cognitive styles' of the men who produced and purchased these trays or those of the women who originally used them ", p. 558.

109. Le Zardino de Oration, nous dit-il, a été écrit au milieu du siècle précisément pour des jeunes filles, Michael BAXANDALL, p. 73.

110. Pour ne prendre qu'un exemple les Méditations sur la Vie du Christ, long attribuées à Bonaventure, étaient écrites pour une lectrice.

111. Olivia POWELL, p 131. n. 14 et Patricia EMISON, p. 430.

112. Baldassare CASTIGLIONE, Le livre du courtisan [1528], trad. A. Pons d'après la version de G. Chappuis, Paris, GF-Flammarion, 1991, livre III.

113. Thomas GOLSENNE, p. 142.

114. Christiane KLAPISCH-ZUBER, « Les saintes poupées: jeu et dévotion dans la Florence du Quattrocento», in J.-C. Margolin et Phillippe. Ariès (éd.), Les jeux à la Renaissance, Actes du XXIIIe colloque international d'études humanistes (Tours, juillet 1980), Paris, 1983, p. 65-79.

115. Ibid., p. 66.

116. Ibid.

117. Ibid.

118. Ibid.

119. Ibid., p. 68 .

120. Christiane KLAPISCH-ZUBER, p. 67.

121. Dans le monde méditerranéen plus large, de Venise en Catalogne, les femmes de l'élite rechangent des statuettes de la Vierge, et s'occupent de sa toilette ; voir Marlène ALBERT-LLORCA, « La Vierge mise à nu par ses chambrières », Clio. Histoire, femmes et sociétés [En ligne], 2 | 1995, mis en ligne le 01 janvier 2005, consulté le 27 septembre 2019. URL: http:// journals.openedition.org/clio/494 ; DOI : 10.4000/clio.494 
122. Ibid., p. 16.

123. Annick DELFOSSE, «Vêtir la Vierge : une grammaire identitaire ", Quand l'habit faisait le moine. Une histoire du vêtement civil et religieux en Luxembourg et au-delà, éd.Olivier Donneau, Bastogne, Musée en Piconrue, 2004, p. 199-208 (p. 205).

124. Richard TREXLER, Public Life in Renaissance Florence, Ithaca, Cornelle University press, 1980, 1991 p. 361.

125. BonAVEnTURE, Lignum Vitae, éd. Jacques Guy Bougerol, Paris, Éditions franciscaines, 1996, p. 62-64, dans un discours adressé à la Vierge : « aspice illam dilecti Filii tui sacratissimam vestem de castissimis membris tuis Spiriti Sancti artificiositate contextam et una cum ipso confugientibus nobilis ad te veniamm postula ».

126. PAUL, Épitre aux Éphésiens, 4,22-24 dit: "On vous a enseigné à vous débarrasser du vieil homme qui correspond à votre ancienne manière de vivre et se détruit sous l'effet de ses désirs trompeurs, à vous laisser renouveler par l'Esprit dans votre intelligence et à vous revêtir de l'homme nouveau, créé selon Dieu dans la justice et la sainteté que produit la vérité. » (« deponere vos secundum pristinam conversationem veterem hominem, qui corrumpitur secundum desideria erroris. Renovamini autem spiritu mentis vestrae, et induite novum hominem, qui secundum Deum creatus est in justitia, et sanctitate veritatis»). et Épitre aux Colossiens 3,9-11: "vous étant dépouillés du vieil homme et de ses oeuvres,et ayant revêtu l'homme nouveau, qui se renouvelle, dans la connaissance, selon l'image de celui qui l'a créé »(« expoliantes vos veterem hominem cum actibus suis et induentes novum eum, qui renovatur in agnitionem secundum imaginem ejus qui creavit illum »).

127. Pour ne prendre qu'un exemple, encore de BONAVENTURE, le corps du Christ crucifié est si sanguinolent « qu'il apparut drapé de rouge et vêtu comme un fouleur au pressoir [...] sa tunique teinte dans le sang [...] semblable à la chair du péché (Lignum Vitae, p. 62-63: «quatenus vere rubrum appareret indumentum ipsius, et vestimenta eius quasi calcantium in torculari. Et sic, vero Ioseph in veterem dimisso cisternam, tunica ipsius intincta sanguine hoedi, propter similitudinem scilicet carnis peccati [...] Hoc est enim Domine vestimentum quod in man meretricis Aegyptiae, synagoga videlicet, innocens Puer tuus sponte dimisit »). Pour plus sur l'anthropologie corps-étoffe voir Alain BOUREAU, De vagues individus La condition humaine dans la pensée scolastique, Paris, Les Belles Lettres, 2008.

128. B. CASTIGLIONE, livre III, VIII, p. 239-40.

129. Pour l'association entre Marie Madeleine et la vanité voir les pages de Katherine LUDWIG JANSEN, p. 145-167.

130. Elisa TOSI BRANDI «Fashion,Art,History and Society in Portraits of Women by Piero del Pollaiuolo » in Antonio et Piero Pollaiolo : nel argento et nel oro, in pittura et nel bronzo... Milan, Skira, 2014, p. 103-117.

131. «[...] e come si maritò gli tagliò una cotta di zetani chermisi ; e così la roba di quello medesimo : ed è 'l più bel drappo qui sia in Firenze; che se lo fece in bottega. E fassi una grillanda di penne con perle, che viene fiorini ottanta; l'acconciatura di sott, e' sono duo trecce di perle, che viene fiorini sessanta $o$ più : che quandro andrà fuori arà in dosso più che fiorini quattrocento. E ordina di fare un velluto chermisi, perfarlo colle maniche grandi, foderato di martore, quando n'andrà a marito: e fa una cioppa rosata, ricamata di perle " (Alessandra MACINGHI NEGLI STROZZI, Lettere di una gentildonna fiorentina del secolo XVai figliuoli esuli, Florence, Sansoni, 1877, p. 5-6.

132. Christiane KLAPISCH-ZUBER explore cet investissement financier dans le vêtement, dans « Le complexe de Griselda » voir note 18.

133. Carole COLLIER FRICK, "Gendered Space in Renaissance Florence: Theorizing Public and Private in the "Rag Trade", » Fashion Theory, 9:2, 2005, p. 125-145.

134. Adrian Randolph nuance pourtant cette idée en analysant un regard féminin dans une autre œuvre de Crivelli, Adrian W. B. RANDOLPH, «Les seuils de l'expérience. L'Annonciation de Crivelli et le genre de la peinture », Perspective [En ligne], 4 | 2007, Publié le 31 mars 2018, consulté le 26 
septembre 2019. URL: http://journals.openedition.org/perspective/3572； DOI: 10.4000/ perspective. 3572

135. Olivia POWELL, p. 63.

136. Sharon FERMOR, "On the Question of Pictorial 'Evidence' for Fifteenth-Century Dance Technique ", Dance Research: The Journal of the Society for Dance Research, Vol. 5, No. 2 (Autumn, 1987), p. 18-32.

137. Caterina LAWLESS « Sensing the Image: Gender, Piety, and Images in Late Medieval Tuscany, Open Arts Journal, Issue 4, Winter, 2014-2015, p. 61-74, approfondit la notion de cinq sens et particulièrement la tactilité dans l'expérience de l'image médiévale pour la femme.

138. La grande pionnière de ces études est Caroline WALKER BYNUM, Jeûnes et festins sacrés: les femmes et la nourriture dans la spiritualité médiévale, tr. Claire Forestier-Pergnier, et. al., Paris, Cerf, 1994 ; Fragmentation and Redemption. Essays on Gender and the Human Body in Medieval Religion, édition révisée, New York / Cambridge, Mass., Zone Books, 1992.

139. Adrian W. B. RANDOLPH, « Deschi », p. 552.

140. Patricia EMISON, « Grazia », Renaissance Studies · Volume 5, Issue 4 , 1991, p. 430. Pour plus sur ce point, voir Simona BRAMBILLA, «Ritratti femminili nelle lettere di Alessandra Macinghi Strozzi », Arzanà [En ligne], $20 \mid 2019$, mis en ligne le 23 juillet 2019, consulté le 26 septembre 2019. URL : http://journals.openedition.org/arzana/1240 ; DOI : 10.4000/arzana.1240

141. Rona GOFFEn, Titian's Women, Yale University Press, New Haven, Londres, 1997, p. 152-159

142. Adrian W. B. RANDOLPH «Deschi... », p. 553-554.

143. Vasari parle de la Madeleine dans l'Extase de Sainte Cécile, autre œuvre d'une commande féminine, de sa pose en tant que «leggiadrissim $[a]$ », lorsque le thème de la musique domine la figure centrale. Giorgio VASARI, "Vie de Raphaël d'Urbin, peintre et architecte », dans Vie des meilleurs peintres, sculpteurs et architectes, éd. et trad. dirigée par André Chastel, Paris, BergerLevrault, 1981-1987, t. 5, p. 211.

144. Dans son Traité de la Peinture, cité et traduit par Daniel ARASSE, Léonard de Vinci, Paris, Hazan, 1997, p. 465.

145. Ibid., Leonard de Vinci, p. 465.

\section{RÉSUMÉS}

Les deux Marie-Madeleine peintes par Carlo Crivelli pour les franciscains à la fin $d u \mathrm{xv}^{\mathrm{e}}$ siècle, conservées aujourd'hui à Montefiore dell'Aso et à Amsterdam, font partie des œuvres les plus connues du peintre vénéto-marchisan. Leurs grâces maniérées, leur mise élégante et élaborée, et leur regard en coin intriguent le spectateur qui pourrait s'attendre à une mise en scène différente d'une prostituée pénitente. Cet article montre comment ces choix sont reliés significativement aux traditions dévotionnelles et artistiques de Venise et des Marches, spécifiquement dans un contexte franciscain, mais aussi comment ils véhiculent notamment des idées sur Marie-Madeleine propres à la période, vue moins comme une pénitente que comme une sainte séductrice. J'examine pendant la discussion la façon dont le parfum de la Madeleine est dépeint, et je porte une attention particulière à la possibilité de peindre la danse dans la Madeleine d'Amsterdam, tout en explorant la notion du regard genré. 
The two Mary Magdalens that Carlo Crivelli painted for the Franciscans in the latter 15th C, now at Montefiore del'Aso and Amsterdam, are some of the most popular works of the VenetoMarchesan painter. Their mannered graces, elegant and elaborate array, and sidelong glances intrigue the viewer who might expect a different appearance from a penitent prostitute. This paper examines how these choices are meaningful within the artistic and devotional traditions of Venice and the Marches, specifically within a Franciscan context, but also how they bear specifically on the period notions of Mary Magdalen, less as penitent than as a sort of sainted seductress. In the course of my argument, I examine how the perfume of the the Magdalen is pictured, with special attention to the possibility of picturing danse with regard to the Rijksmuseum image, while exploring the notion of the gendered gaze.

\section{INDEX}

Keywords : Franciscan art, venetian art, marchisan art, danse, perfume, gendered gaze, Carlo Crivelli, Mary Magdelen, courtesan, dressing, holy dolls

Mots-clés : art franciscain, art vénitien, art marchisan danse, parfum, regard genré, Carlo Crivelli, Marie Madeleine, courtisane, habillement, poupées saintes, tactilité

\section{AUTEUR}

\section{VICKI-MARIE PETRICK}

Vicki-Marie Petrick a soutenu sa thèse, Le Corps de Marie-Madeleine et ses représentations en Italie du Duecento à Titien, à l'EHESS en 2012 - commencée sous Daniel Arasse et finie sous Sylvain Pironavec mention honorable et félicitations du jury. Elle continue ses recherches dans ce domaine, s'intéressant particulièrement sur l'art franciscain, l'art vénitien, l'alchimie, et le genre, et le matière de la peinture, et les sibylles, entre autre. Elle enseigne l'histoire de l'art à Schiller International University. 